\title{
Variational linearization of pure traction problems in incompressible elasticity
}

Edoardo Mainini(D) and Danilo Percivale

Abstract. We consider pure traction problems, and we show that incompressible linearized elasticity can be obtained as variational limit of incompressible finite elasticity under suitable conditions on external loads.

Mathematics Subject Classification. 49J45, 74K30, 74K35, 74R10.

Keywords. Calculus of variations, Linear elasticity, Finite elasticity, Gamma-convergence, Rubber-like materials.

\section{Introduction}

Let us consider a hyperelastic body occupying a bounded open set $\Omega \subset \mathbb{R}^{3}$ in its reference configuration. Equilibrium states under a body force field $\mathfrak{f}: \Omega \rightarrow \mathbb{R}^{3}$ and a surface force field $\mathfrak{g}: \partial \Omega \rightarrow \mathbb{R}^{3}$ are obtained by minimizing the total energy

$$
\int_{\Omega} \mathcal{W}^{I}(x, \nabla \mathbf{y}(x)) \mathrm{d} x-\int_{\Omega}(\mathbf{y}(x)-x) \cdot \mathfrak{f}(x) \mathrm{d} x-\int_{\partial \Omega}(\mathbf{y}(x)-x) \cdot \mathfrak{g}(x) \mathrm{d} \mathcal{H}^{2}(x) .
$$

Here, $\mathbf{y}: \Omega \rightarrow \mathbb{R}^{3}$ denotes the deformation field, $\mathcal{H}^{2}$ denotes the surface measure, and $\mathcal{W}^{I}: \Omega \times \mathbb{R}^{3 \times 3} \rightarrow$ $[0,+\infty]$ is the incompressible strain energy density. We require incompressibility by letting $\mathcal{W}^{I}(x, \mathbf{F})=$ $+\infty$ whenever $\operatorname{det} \mathbf{F} \neq 1$. Moreover, we assume that $\mathcal{W}^{I}(x, \cdot)$ is a frame indifferent function that is minimized at $\mathbf{F}=\mathbf{I}$ with value 0 .

If $h>0$ is an adimensional small parameter, we rescale the displacement field and the external forces by letting $\mathfrak{f}=h \mathbf{f}, \mathfrak{g}=h \mathbf{g}$ and $\mathbf{y}(x)-x=h \mathbf{v}(x)$. We get

$$
\mathcal{E}_{h}^{I}(\mathbf{v}):=\int_{\Omega} \mathcal{W}^{I}(x, \mathbf{I}+h \nabla \mathbf{v}) \mathrm{d} x-h^{2} \int_{\Omega} \mathbf{f} \cdot \mathbf{v} \mathrm{d} x-h^{2} \int_{\partial \Omega} \mathbf{g} \cdot \mathbf{v} \mathrm{d} \mathcal{H}^{2}(x) .
$$

We aim at obtaining the behavior of rescaled energies as $h \rightarrow 0$ and at showing that the linearized elasticity functional arises in the limit. More precisely, we aim at proving that

$$
\inf \mathcal{E}_{h}^{I}=h^{2} \min \mathcal{E}^{I}+o\left(h^{2}\right),
$$

and that if $\mathcal{E}_{h}^{I}\left(\mathbf{v}_{h}\right)-\inf \mathcal{E}_{h}^{I}=o\left(h^{2}\right)$ (i.e., if $\mathbf{v}_{h}$ is a sequence of almost minimizers for $\mathcal{E}_{h}^{I}$ ) then

$$
\mathbf{v}_{h} \rightarrow \mathbf{v}_{0} \in \operatorname{argmin} \mathcal{E}^{I}
$$

in a suitable sense, where

$$
\mathcal{E}^{I}(\mathbf{v}):=\int_{\Omega} \mathcal{Q}^{I}(x, \mathbb{E}(\mathbf{v})) \mathrm{d} x-\int_{\Omega} \mathbf{f} \cdot \mathbf{v} \mathrm{d} x-\int_{\partial \Omega} \mathbf{g} \cdot \mathbf{v} \mathrm{d} \mathcal{H}^{2}(x) .
$$


Here, $\mathbb{E}(\mathbf{v}):=\frac{1}{2}\left(\nabla \mathbf{v}+\nabla \mathbf{v}^{T}\right)$ is the infinitesimal strain tensor field and $\mathcal{Q}^{I}(x, \cdot)$ is defined for every $\mathbf{F} \in \mathbb{R}^{3 \times 3}$ by

$$
\mathcal{Q}^{I}(x, \mathbf{F}):=\lim _{h \rightarrow 0} h^{-2} \mathcal{W}^{I}(x, \exp (h \mathbf{F}))= \begin{cases}\frac{1}{2} \mathbf{F}^{T} D^{2} \mathcal{W}(x, \mathbf{I}) \mathbf{F} & \text { if } \operatorname{Tr} \mathbf{F}=0 \\ +\infty & \text { if } \operatorname{Tr} \mathbf{F} \neq 0\end{cases}
$$

where $\mathcal{W}(x, \mathbf{F}):=\mathcal{W}^{I}\left(x,(\operatorname{det} \mathbf{F})^{-1 / 3} \mathbf{F}\right)$ is the isochoric part of $\mathcal{W}^{I}$. Such a quadratic form is obtained by a formal Taylor expansion around the identity matrix ( $D^{2}$ denoting the Hessian in the second variable). Since $\mathcal{Q}^{I}(x, \mathbf{F})=+\infty$ if $\operatorname{Tr} \mathbf{F} \neq 0$, we see that $\mathcal{E}^{I}(\mathbf{v})$ is finite only if $\operatorname{div} \mathbf{v}=0$ a.e. in $\Omega$. Therefore, $\mathcal{E}^{I}$ is the linearized elastic energy with elasticity tensor $D^{2} \mathcal{W}(x, \mathbf{I})$ and $\operatorname{div} \mathbf{v}=0$ is the linearized incompressibility constraint.

Under Dirichlet boundary conditions, (1.1)-(1.2) have been obtained in [25], by means of a $\Gamma$-convergence analysis with respect to the weak topology of $W^{1, p}\left(\Omega, \mathbb{R}^{3}\right)$, where the exponent $p$ is suitably related to the coercivity properties of $\mathcal{W}^{I}$ (see Sect. 2). On the other hand, in this paper we shall consider natural Neumann boundary conditions, i.e., the pure traction problem. In this case, it is crucial to impose suitable restrictions on the external forces. In particular, as done in [23,24], here we shall assume they have null resultant and null momentum with respect to the origin, namely

$$
\mathbb{E}(\mathbf{v})=0 \Rightarrow \int_{\partial \Omega} \mathbf{g} \cdot \mathbf{v} d \mathcal{H}^{2}(x)+\int_{\Omega} \mathbf{f} \cdot \mathbf{v} \mathrm{d} x=0,
$$

and that they satisfy the following strict compatibility condition

$$
\int_{\partial \Omega} \mathbf{g} \cdot \mathbf{W}^{2} x d \mathcal{H}^{2}(x)+\int_{\Omega} \mathbf{f} \cdot \mathbf{W}^{2} x \mathrm{~d} x<0 \quad \forall \mathbf{W} \in \mathbb{R}_{\text {skew }}^{3 \times 3}, \quad \mathbf{W} \neq \mathbf{0},
$$

where $\mathbb{R}_{\text {skew }}^{3 \times 3}$ denotes the set of real $3 \times 3$ skew-symmetric matrices. For suitable classes of external forces, the latter condition can be interpreted as an overall dilation effect on the body, see Remark 2.7 later on. Even under such restrictions, in this case it is not possible to obtain a sequential $\Gamma$-convergence result with respect to the weak convergence in $W^{1, p}\left(\Omega, \mathbb{R}^{3}\right)$ or to the weak $L^{p}\left(\Omega, \mathbb{R}^{3 \times 3}\right)$ convergence of infinitesimal strain tensors (we stress that the elastic part in $\mathcal{E}_{h}^{I}$ is not invariant by infinitesimal rigid displacements, see also Remark 2.6). However, in this context we will show that $\mathcal{E}^{I}$ provides indeed an upper bound for the sequence $h^{-2} \mathcal{E}_{h}^{I}\left(\mathbf{v}_{h}\right)$ in the limit $h \rightarrow 0$, as soon as $\mathbb{E}\left(\mathbf{v}_{h}\right) \rightarrow \mathbb{E}(\mathbf{v})$ weakly in $L^{p}\left(\Omega, \mathbb{R}^{3 \times 3}\right)$ and $\operatorname{det}\left(\mathbf{I}+h \nabla \mathbf{v}_{h}\right)=1$. On the other hand, due to the lack of control on skew-symmetric parts, we may only obtain a lower bound in terms of the functional

$$
\mathcal{F}^{I}(\mathbf{v}):=\min _{\mathbf{W} \in \mathbb{R}_{\text {skew }}^{3 \times 3}} \int_{\Omega} \mathcal{Q}^{I}\left(x, \mathbb{E}(\mathbf{v})-\frac{1}{2} \mathbf{W}^{2}\right) \mathrm{d} x-\int_{\Omega} \mathbf{f} \cdot \mathbf{v} \mathrm{d} x-\int_{\partial \Omega} \mathbf{g} \cdot \mathbf{v} \mathrm{d} \mathcal{H}^{2}(x) .
$$

Clearly, we have $\mathcal{F}^{I} \leq \mathcal{E}^{I}$. Interestingly, this still allows to obtain (1.1)-(1.2), since it is possible to show (see Lemma 7.1 later on) that if $\mathbf{v} \in \operatorname{argmin} \mathcal{F}^{I}$, then $\mathcal{F}^{I}(\mathbf{v})=\mathcal{E}^{I}(\mathbf{v})$. Indeed, if $\mathbf{v} \in \operatorname{argmin} \mathcal{F}^{I}$, then the minimization problem inside the definition of $\mathcal{F}^{I}$ is solved by $\mathbf{W}=0$.

A key step for obtaining the proof of (1.1)-(1.2) will be to approximate divergence-free vector fields in terms of vector fields $\mathbf{v}_{t}$ having the property $\mathcal{W}^{I}\left(x, \mathbf{I}+t \nabla \mathbf{v}_{t}\right)<+\infty$, i.e., $\operatorname{det}\left(\mathbf{I}+t \nabla \mathbf{v}_{t}\right)=1$ for any $t>0$. Following the approach of [25], we define

$$
\mathbf{v}_{t}(x):=\frac{\mathbf{y}_{t}(x)-x}{t}
$$

where $\mathbf{y}_{t}$ is the flow associated to the divergence-free field $\mathbf{v}$, starting from $\mathbf{y}_{0}(x)=x \in \Omega$, and then by Reynolds transport formula we see that $\mathbf{y}_{t}$ is a volume preserving deformation field. Indeed, for any $A \subseteq \Omega$ we have

$$
\frac{\mathrm{d}}{\mathrm{d} t}\left|\mathbf{y}_{t}(A)\right|=\int_{A} \operatorname{div} \mathbf{v}(x) \mathrm{d} x=0
$$


so that $\operatorname{det} \nabla \mathbf{y}_{t}=\operatorname{det}\left(\mathbf{I}+t \nabla \mathbf{v}_{t}\right)=1$. In this paper, we will further develop this approach in order to obtain the desired recovery sequence and the upper bound under the above conditions on external loads. Moreover, since we have natural boundary conditions, we avoid the technical difficulties due to the need of keeping track of the boundary data through the construction of the recovery sequence. Therefore, we shall not need the strong regularity assumptions on $\partial \Omega$ that were imposed in [25].

Hyperelastic incompressible models are typically used to describe rubber-like materials such as artificial elastomers as well as biological soft tissues $[9,16,17,19,28,30,36,40]$. We refer to $[5-7,26,39,41]$ for many examples of strain energy densities that are used for the nonlinear description of the stress-strain behavior of these materials. On the other hand, linear modeling is usually considered a good approximation in the small strain regime. Indeed, the classical theory of linearized elasticity is based on the smallness assumption on deformation gradients, see for instance [14,22,38]. Nevertheless, for a variational derivation, i.e., for the proof of (1.1)-(1.2), no a priori smallness assumption is needed, leading to a rigorous justification of linearized elasticity (we also stress that small loads need not give rise to small strains in rubber-like materials, due to their high compliance in shear). The first rigorous variational derivation of linearized elasticity from finite elasticity is given in [11], where $\Gamma$-convergence and convergence of minimizers of the associated Dirichlet boundary value problems are proven in the compressible case. We refer to $[1-3,10,18,23-25,37]$ for many other results of this kind, some of which including theories for incompressible materials $[10,18,25]$. The study of asymptotic properties of minimal energies, similar to (1.1)-(1.2), is also typical of dimension reduction problems, see for instance [4,21,31-35].

In the next section, we rigorously state the main theorem, providing the proof of (1.1)-(1.2) for the pure traction problem. A related result has been recently obtained by Jesenko and Schmidt in [18] under different assumptions on the external loads, but in the more general framework of multiwell potentials that leads to a suitable quasiconvex envelope of the strain energy density in the limiting functional.

\section{Plan of the paper}

In Sect. 2, we collect the assumptions of the theory and we state the main result about convergence of minimizers. The latter is based on suitable compactness properties of (almost) minimizing sequences that are established in Sect. 4, after some preliminaries in Sect. 3. In Sect. 5, we provide the lower bound. Sect. 6 delivers the upper bound. Section 7 completes the proof of the main result.

\section{Main result}

In this section, we introduce all the assumptions and we state the main result. Let $\Omega \subset \mathbb{R}^{3}$ be the reference configuration of the body. We assume that for some $m \in \mathbb{N}$

$\Omega$ is a bounded open connected Lipschitz set, $\partial \Omega$ has $m$ connected components.

\section{Assumptions on the elastic energy density}

We let $\mathcal{W}^{I}: \Omega \times \mathbb{R}^{3 \times 3} \rightarrow[0,+\infty]$ be $\mathcal{L}^{3} \times \mathcal{B}^{9}$ - measurable. The assumptions on $\mathcal{W}^{I}$ are similar to the ones in $[2,25]$, i.e., for a.e. $x \in \Omega$

$$
\begin{gathered}
\mathcal{W}^{I}(x, \mathbf{F})=+\infty \quad \text { if } \operatorname{det} \mathbf{F} \neq 1, \\
\mathcal{W}^{I}(x, \mathbf{R F})=\mathcal{W}^{I}(x, \mathbf{F}) \quad \forall \mathbf{R} \in S O(3), \quad \forall \mathbf{F} \in \mathbb{R}^{3 \times 3},
\end{gathered}
$$




$$
\min \mathcal{W}^{I}=\mathcal{W}^{I}(x, \mathbf{I})=0 .
$$

Moreover, we define $\mathcal{W}: \Omega \times \mathbb{R}^{3 \times 3} \rightarrow[0,+\infty]$ by

$$
\mathcal{W}(x, \mathbf{F}):= \begin{cases}\mathcal{W}^{I}\left(x,(\operatorname{det} \mathbf{F})^{-1 / 3} \mathbf{F}\right) & \text { if } \operatorname{det} \mathbf{F}>0 \\ +\infty & \text { if } \operatorname{det} \mathbf{F} \leq 0\end{cases}
$$

and we assume that $\mathcal{W}(x, \cdot)$ is $C^{2}$ in a neighbor of rotations, i.e.,

there exists a neighborhood $\mathcal{U}$ of $S O(3)$ s.t., for a.e. $x \in \Omega, \mathcal{W}(x, \cdot) \in C^{2}(\mathcal{U})$,

with a modulus of continuity of $D^{2} \mathcal{W}(x, \cdot)$ that does not depend on $x$.

Moreover, there exists $K>0$ such that $\left|D^{2} \mathcal{W}(x, \mathbf{I})\right| \leq K$ for a.e. $x \in \Omega$.

$(\mathcal{W} 3)$

We assume the following growth property from below: there exist $C>0$ and $p \in(1,2]$ such that for a.e. $x \in \Omega$

$$
\mathcal{W}^{I}(x, \mathbf{F}) \geq C g_{p}(d(\mathbf{F}, S O(3))) \quad \forall \mathbf{F} \in \mathbb{R}^{3 \times 3},
$$

where $g_{p}:[0,+\infty) \rightarrow \mathbb{R}$ is the convex function defined by

$$
g_{p}(t)= \begin{cases}t^{2} & \text { if } 0 \leq t \leq 1 \\ \frac{2 t^{p}}{p}-\frac{2}{p}+1 & \text { if } t \geq 1\end{cases}
$$

Here, $S O(3)$ denotes the group of positive rotation matrices. $d(\cdot, S O(3))$ denotes the distance from $S O(3)$ and it satisfies

$$
d(\mathbf{F}, S O(3)):=\inf _{\mathbf{R} \in S O(3)}|\mathbf{F}-\mathbf{R}|=\left|\sqrt{\mathbf{F}^{T} \mathbf{F}}-\mathbf{I}\right| \quad \text { for any } \mathbf{F} \in \mathbb{R}^{3 \times 3} \text { with } \operatorname{det} \mathbf{F}>0
$$

where $|\cdot|$ is the Euclidean norm on $\mathbb{R}^{3 \times 3}$, i.e., $|\mathbf{F}|:=\sqrt{\operatorname{Tr}\left(\mathbf{F}^{T} \mathbf{F}\right)}$.

\section{Assumptions on the external forces}

We introduce a body force field $\mathbf{f} \in L^{\frac{3 p}{4 p-3}}\left(\Omega, \mathbb{R}^{3}\right)$ and a surface force field $\mathbf{g} \in L^{\frac{2 p}{3 p-3}}\left(\partial \Omega, \mathbb{R}^{3}\right)$, where $p$ is such that $(\mathcal{W} 4)$ holds. The corresponding contribution to the energy is given by the linear functional

$$
\mathcal{L}(\mathbf{v}):=\int_{\Omega} \mathbf{f} \cdot \mathbf{v} \mathrm{d} x+\int_{\partial \Omega} \mathbf{g} \cdot \mathbf{v} d \mathcal{H}^{2}(x), \quad \mathbf{v} \in W^{1, p}\left(\Omega, \mathbb{R}^{3}\right) .
$$

We note that since $\Omega$ is a bounded Lipschitz domain, the Sobolev embedding $W^{1, p}\left(\Omega, \mathbb{R}^{3}\right) \hookrightarrow$ $L^{\frac{3 p}{3-p}}\left(\Omega, \mathbb{R}^{3}\right)$ and the Sobolev trace embedding $W^{1, p}\left(\Omega, \mathbb{R}^{3}\right) \hookrightarrow L^{\frac{2 p}{3-p}}\left(\partial \Omega, \mathbb{R}^{3}\right)$ imply that $\mathcal{L}$ is a bounded functional over $W^{1, p}\left(\Omega, \mathbb{R}^{3}\right)$.

We assume that external loads have null resultant and null momentum, i.e.,

$$
\mathbb{E}(\mathbf{v})=0 \Rightarrow \mathcal{L}(\mathbf{v})=0,
$$

and that they satisfy the following strict compatibility condition

$$
\mathcal{L}\left(\mathbf{W}^{2} x\right)<0 \quad \forall \mathbf{W} \in \mathbb{R}_{\text {skew }}^{3 \times 3}, \mathbf{W} \neq \mathbf{0} .
$$

Some examples of external loads satisfying the above assumptions are provided in the remarks at the end of this section. 


\section{Statement of the main result}

The functional representing the scaled total energy is denoted by $\mathcal{F}_{h}^{I}: W^{1, p}\left(\Omega, \mathbb{R}^{3}\right) \rightarrow \mathbb{R} \cup\{+\infty\}$ and defined as follows

$$
\mathcal{F}_{h}^{I}(\mathbf{v}):=\frac{1}{h^{2}} \int_{\Omega} \mathcal{W}^{I}(x, \mathbf{I}+h \nabla \mathbf{v}) \mathrm{d} x-\mathcal{L}(\mathbf{v}) .
$$

We further introduce the functional of linearized incompressible elasticity $\mathcal{E}^{I}: W^{1, p}\left(\Omega, \mathbb{R}^{3}\right) \rightarrow \mathbb{R} \cup$ $\{+\infty\}$ as

$$
\mathcal{E}^{I}(\mathbf{v}):= \begin{cases}\int_{\Omega} \mathcal{Q}^{I}(x, \mathbb{E}(\mathbf{v})) \mathrm{d} x-\mathcal{L}(\mathbf{v}) & \text { if } \mathbf{v} \in H^{1}\left(\Omega, \mathbb{R}^{3}\right) \\ +\infty & \text { otherwise in } W^{1, p}\left(\Omega, \mathbb{R}^{3}\right)\end{cases}
$$

where

$$
\mathcal{Q}^{I}(x, \mathbf{B}):= \begin{cases}\frac{1}{2} \mathbf{B}^{T} D^{2} \mathcal{W}(x, \mathbf{I}) \mathbf{B} & \text { if } \operatorname{Tr} \mathbf{B}=0 \\ +\infty & \text { otherwise }\end{cases}
$$

and the limit energy functional $\mathcal{F}^{I}: W^{1, p}\left(\Omega, \mathbb{R}^{3}\right) \rightarrow \mathbb{R} \cup\{+\infty\}$ defined by

$$
\mathcal{F}^{I}(\mathbf{v}):= \begin{cases}\inf _{\mathbf{w} \in \mathbb{R}_{\text {skew }}^{3 \times 3}} \int_{\Omega} \mathcal{Q}^{I}\left(x, \mathbb{E}(\mathbf{v})-\frac{\mathbf{W}^{2}}{2}\right) \mathrm{d} x-\mathcal{L}(\mathbf{v}) & \text { if } \mathbf{v} \in H^{1}\left(\Omega, \mathbb{R}^{3}\right) \\ +\infty & \text { otherwise in } W^{1, p}\left(\Omega, \mathbb{R}^{3}\right) .\end{cases}
$$

We notice that $\mathcal{E}^{I}(\mathbf{v})$ is finite only if $\mathbf{v} \in H^{1}\left(\Omega, \mathbb{R}^{3}\right)$ is divergence-free, while $\mathcal{F}^{I}(\mathbf{v})$ is finite only if $\mathbf{v}$ has constant nonpositive divergence (since $\mathbf{W}^{2}$ is negative semi-definite for any $\mathbf{W} \in \mathbb{R}_{\text {skew }}^{3 \times 3}$ ).

We are ready for the statement of the main result

Theorem 2.1. Assume $(2.1),(\mathcal{L} 1),(\mathcal{L} 2),(\mathcal{W} \mathbf{0}),(\mathcal{W} 1),(\mathcal{W} \mathbf{2}),(\mathcal{W} 3),(\mathcal{W} 4)$. Then, for every vanishing sequence $\left(h_{j}\right)_{j \in \mathbb{N}} \subset(0,1)$ we have

$$
\inf _{W^{1, p}\left(\Omega, \mathbb{R}^{3}\right)} \mathcal{F}_{h_{j}}^{I} \in \mathbb{R}
$$

If $\left(\boldsymbol{v}_{j}\right)_{j \in \mathbb{N}} \subset W^{1, p}\left(\Omega, \mathbb{R}^{3}\right)$ is a sequence such that

$$
\lim _{j \rightarrow+\infty}\left(\mathcal{F}_{h_{j}}^{I}\left(\mathbf{v}_{j}\right)-\inf _{W^{1, p}\left(\Omega, \mathbb{R}^{3}\right)} \mathcal{F}_{h_{j}}^{I}\right)=0,
$$

then there is a (not relabeled) subsequence such that

$$
\mathbb{E}\left(\mathbf{v}_{j}\right) \rightarrow \mathbb{E}\left(\mathbf{v}_{*}\right) \text { weakly in } L^{p}\left(\Omega, \mathbb{R}^{3}\right) \quad \text { as } j \rightarrow+\infty,
$$

where $\mathbf{v}_{*} \in H^{1}\left(\Omega, \mathbb{R}^{3}\right)$ is a minimizer of $\mathcal{F}^{I}$ over $W^{1, p}\left(\Omega, \mathbb{R}^{3}\right)$, and

$$
\mathcal{F}_{h_{j}}^{I}\left(\mathbf{v}_{j}\right) \rightarrow \mathcal{F}^{I}\left(\mathbf{v}_{*}\right)=\mathcal{E}^{I}\left(\mathbf{v}_{*}\right), \quad \inf _{W^{1, p}\left(\Omega, \mathbb{R}^{3}\right)} \mathcal{F}_{h_{j}}^{I} \rightarrow \min _{W^{1, p}\left(\Omega, \mathbb{R}^{3}\right)} \mathcal{F}^{I}=\min _{W^{1, p}\left(\Omega, \mathbb{R}^{3}\right)} \mathcal{E}^{I} \quad \text { as } j \rightarrow+\infty .
$$

We close this section with several remarks about the main theorem. 
Remark 2.2. It is worth noticing that the infimum in the right-hand side of (2.4) is actually a minimum. Indeed if $\mathbf{v} \in H^{1}\left(\Omega, \mathbb{R}^{3}\right)$ then either $\mathcal{F}^{I}(\mathbf{v})=+\infty$ or $\operatorname{div} \mathbf{v}$ is a nonpositive constant. In the latter case, let $\left(\mathbf{W}_{n}\right)_{n \in \mathbb{N}} \subset \mathbb{R}_{\text {skew }}^{3 \times 3}$ be a minimizing sequence: then $\left|\mathbf{W}_{n}\right|^{2}=\operatorname{Tr}\left(\mathbf{W}_{n}^{T} \mathbf{W}_{n}\right)=-\operatorname{Tr} \mathbf{W}_{n}^{2}=-2 \operatorname{div} \mathbf{v}$ hence there exists $\mathbf{W}_{\mathbf{v}}$ such that, up to subsequences, $\mathbf{W}_{n} \rightarrow \mathbf{W}_{\mathbf{v}}$ and $\mathcal{F}^{I}(\mathbf{v})=\int_{\Omega} \mathcal{Q}^{I}\left(\mathbb{E}(\mathbf{v})-\frac{\mathbf{W}_{\mathbf{v}}^{2}}{2}\right) \mathrm{d} x-\mathcal{L}(\mathbf{v})$ as claimed.

Remark 2.3. If the function $\mathcal{W}$ is replaced by any other $\widetilde{\mathcal{W}}$, still satisfying $(\mathcal{W} \mathbf{3})$, such that $\mathcal{W}(x, \mathbf{F})=$ $\widetilde{\mathcal{W}}(x, \mathbf{F})$ if $\operatorname{det} \mathbf{F}=1$, then this does not affect functionals $\mathcal{F}^{I}$ and $\mathcal{E}^{I}$. Indeed, if $\operatorname{Tr} \mathbf{B}=0$ then $\operatorname{det}(\exp (h \mathbf{B}))=\exp (h \operatorname{Tr} \mathbf{B})=1$ and by Taylor's expansion we have for a.e. $x \in \Omega$

$$
\lim _{h \rightarrow 0} \frac{1}{h^{2}} \mathcal{W}^{I}(x, \exp (h \mathbf{B}))=\frac{1}{2} \operatorname{sym} \mathbf{B} D^{2} \widetilde{\mathcal{W}}(x, \mathbf{I}) \operatorname{sym} \mathbf{B}=\frac{1}{2} \operatorname{sym} \mathbf{B} D^{2} \mathcal{W}(x, \mathbf{I}) \operatorname{sym} \mathbf{B}=\mathcal{Q}^{I}(x, \mathbf{B}) .
$$

Remark 2.4. A typical form of $\mathcal{W}^{I}$ is the Ogden incompressible strain energy density, see [8, 16, 29], given by

$$
\mathcal{W}^{I}(\mathbf{F}):=\sum_{k=1}^{N} \frac{\mu_{k}}{\alpha_{k}}\left(\operatorname{Tr}\left(\left(\mathbf{F}^{T} \mathbf{F}\right)^{\alpha_{k} / 2}\right)-3\right), \quad \operatorname{det} \mathbf{F}=1,
$$

where $N, \mu_{k}, \alpha_{k}$ are material constants, and extended to $+\infty$ if $\operatorname{det} \mathbf{F} \neq 1$. If the material constants vary in a suitable range, the Ogden model satisfies the assumptions $(\mathcal{W} \mathbf{1}),(\mathcal{W} \mathbf{2}),(\mathcal{W} \mathbf{3}),(\mathcal{W} \mathbf{4})$. In particular, we refer to [2] and [25] for a discussion about the growth properties and the validity of $(\mathcal{W} 4)$ for the Ogden strain energy density and other standard models.

Remark 2.5. It is worth to stress that Theorem 2.1 does not hold without suitable compatibility assumptions on external forces. Not even relaxing $(\mathcal{L} 2)$ by requiring non strict inequality therein would work. Indeed, choose $\mathbf{f}=\mathbf{g} \equiv 0$,

$$
\mathcal{W}^{I}(\mathbf{x}, \mathbf{F})= \begin{cases}\left|\mathbf{F}^{T} \mathbf{F}-\mathbf{I}\right|^{2} & \text { if } \operatorname{det} \mathbf{F}=1 \\ +\infty & \text { otherwise }\end{cases}
$$

so that $(\mathcal{W} \mathbf{0}),(\mathcal{W} \mathbf{1}),(\mathcal{W} \mathbf{2}),(\mathcal{W} \mathbf{3}),(\mathcal{W} \mathbf{4})$ are satisfied (with $p=2)$. Let $\mathbf{v}_{j}:=h_{j}^{-1}(\mathbf{R}-\mathbf{I}) x$, where $\mathbf{R} \in S O(3), \mathbf{R} \neq \mathbf{I}$ and $\left(h_{j}\right)_{j \in \mathbb{N}} \subset(0,1)$ is a vanishing sequence. Then, $\mathbf{y}_{j}=x+h_{j} \mathbf{v}_{j}=\mathbf{R} x$ hence $\operatorname{det} \nabla \mathbf{y}_{j}=1$ and $\mathcal{F}_{h_{j}}^{I}\left(\mathbf{v}_{j}\right)=0=\min _{W^{1, p}\left(\Omega, \mathbb{R}^{3}\right)} \mathcal{F}_{h_{j}}^{I}$ so the sequence $\left(\mathbf{v}_{j}\right)_{j \in \mathbb{N}}$ satisfies (2.6). However, it has no subsequence that is weakly converging in $W^{1, p}\left(\Omega, \mathbb{R}^{3}\right)$. Moreover, $\mathbb{E}\left(\mathbf{v}_{j}\right)=c h_{j}^{-1} \mathbf{W}^{2}$ for some $c \in(0,1]$ and some $\mathbf{W} \in \mathbb{R}_{\text {skew }}^{3 \times 3}$ such that $|\mathbf{W}|^{2}=2$, as a consequence of the Euler-Rodrigues formula (3.2) for the representation of rotations that we shall recall in Sect. 3 . Therefore, the sequence $\left(\mathbb{E}\left(\mathbf{v}_{j}\right)\right)_{j \in \mathbb{N}}$ has no subsequence that is bounded in $L^{p}\left(\Omega, \mathbb{R}^{3 \times 3}\right)$.

Remark 2.6. Under the assumptions of Theorem 2.1, in general it is not possible to get weak $W^{1, p}\left(\Omega, \mathbb{R}^{3}\right)$ compactness of (almost) minimizing sequences. Indeed, let us consider the following example. Let $\Omega=B_{1}$ be the unit ball of $\mathbb{R}^{3}$, centered at the origin. Let $\mathcal{W}^{I}$ be given by $(2.7)$. Let $\mathbf{f}(x)=x$ and $\mathbf{g} \equiv 0$. It is readily seen that $(\mathcal{L} 1)$ and $(\mathcal{L} 2)$ are satisfied. On the other hand, let the divergence-free vector field $\mathbf{v}_{*} \in H^{1}\left(\Omega, \mathbb{R}^{3}\right)$ be a minimizer of $\mathcal{E}^{I}$ over $W^{1, p}\left(\Omega, \mathbb{R}^{3}\right)$. Since $\Omega=B_{1}$ and $\mathbf{v}_{*}$ is divergence-free, there exists $\mathbf{w}_{*} \in H^{2}\left(\Omega, \mathbb{R}^{3}\right)$ such that $\mathbf{v}_{*}=$ curl $\mathbf{w}_{*}$ and by divergence theorem

$$
\mathcal{L}\left(\mathbf{v}_{*}\right)=-\int_{B_{1}} x \cdot \mathbf{v}_{*} \mathrm{~d} x=-\int_{B_{1}} x \cdot \operatorname{curl} \mathbf{w}_{*} \mathrm{~d} x=\int_{B_{1}} \operatorname{div}\left(x \wedge \mathbf{w}_{*}\right) \mathrm{d} x=\int_{\partial B_{1}}\left(x \wedge \mathbf{w}_{*}\right) \cdot x \mathrm{~d} x=0
$$

so that

$$
\mathcal{E}^{I}\left(\mathbf{v}_{*}\right)=4 \int_{B_{1}}\left|\mathbb{E}\left(\mathbf{v}_{*}\right)\right|^{2} \mathrm{~d} x \geq 0
$$


and since $\mathcal{E}^{I}(\mathbf{0})=0$, we get $\mathcal{E}^{I}\left(\mathbf{v}_{*}\right)=0$. By Theorem 2.1, we deduce that 0 is the minimal value of both $\mathcal{E}^{I}$ and $\mathcal{F}^{I}$ over $W^{1, p}\left(\Omega, \mathbb{R}^{3}\right)$. Let now $\mathbf{W} \in \mathbb{R}_{\text {skew }}^{3 \times 3}$ be such that $|\mathbf{W}|^{2}=2$. Let $\alpha \in\left(\frac{1}{2}, 1\right)$ and let us consider a vanishing sequence $\left(h_{j}\right)_{j \in \mathbb{N}} \subset(0,1)$ and the sequence

$$
\mathbf{v}_{j}(x):=h_{j}^{\alpha-1} \mathbf{W} x+h_{j}^{-1}\left(1-\sqrt{1-h_{j}^{2 \alpha}}\right) \mathbf{W}^{2} x=0 .
$$

The Euler-Rodrigues formula (3.2) implies that for any $j \in \mathbb{N}$ there holds $\mathbf{y}_{j}(x):=x+h_{j} \mathbf{v}_{j}=\mathbf{R}_{j} x$ for a suitable $\mathbf{R}_{j} \in S O(3)$. This implies $\operatorname{det} \nabla \mathbf{y}_{j}=1$ for any $j \in \mathbb{N}$ and then by (2.7) we get

$$
\mathcal{F}_{h_{j}}^{I}\left(\mathbf{v}_{j}\right)=\frac{1}{h_{j}^{2}} \int_{B_{1}} \mathcal{W}\left(x, \mathbf{I}+h_{j} \nabla \mathbf{v}_{j}\right) \mathrm{d} x=\frac{1}{h_{j}^{2}} \int_{B_{1}}\left|2 h_{j} \mathbb{E}\left(\mathbf{v}_{j}\right)+h_{j}^{2} \nabla \mathbf{v}_{j}^{T} \nabla \mathbf{v}_{j}\right|^{2} \mathrm{~d} x .
$$

The above right-hand side goes to 0 as $j \rightarrow+\infty$, as shown after a computation making use of (2.8). Therefore, the sequence $\left(\mathbf{v}_{j}\right)_{j \in \mathbb{N}}$ satisfies (2.6). On the other hand,

$\left(\nabla \mathbf{v}_{j}\right)_{j \in \mathbb{N}}$ has no subsequence that is bounded in $L^{p}\left(\Omega, \mathbb{R}^{3 \times 3}\right)$, since $\alpha \in\left(\frac{1}{2}, 1\right)$.

Remark 2.7. If $\mathcal{L}\left(\mathbf{W}_{*}^{2} x\right)>0$ for some $\mathbf{W}_{*} \in \mathbb{R}_{\text {skew }}^{3 \times 3}$, then the functionals $\mathcal{F}_{h}^{I}$ may admit no uniform bound from below as $h \rightarrow 0$. Indeed, let $\Omega=\left\{\left(x_{1}, x_{2}, x_{3}\right) \in \mathbb{R}^{3}: x_{1}^{2}+x_{2}^{2}<1,0<x_{3}<1\right\}$ and let $\mathbf{g}(x)=$ $\left(-x_{1},-x_{2}, 0\right), \mathbf{f} \equiv 0, \mathbf{W}_{*}:=\mathbf{e}_{1} \otimes \mathbf{e}_{2}-\mathbf{e}_{2} \otimes \mathbf{e}_{1}$. Then, $(\mathcal{L} 1)$ is satisfied and since $\mathbf{W}_{*}^{2}=-\mathbf{e}_{1} \otimes \mathbf{e}_{1}-\mathbf{e}_{2} \otimes \mathbf{e}_{2}$ we get

$$
\mathcal{L}\left(\mathbf{W}_{*}^{2} x\right)=\int_{\partial \Omega}\left(x_{1}^{2}+x_{2}^{2}\right) d \mathcal{H}^{2}(x)>0
$$

and by taking a vanishing sequence $\left(h_{j}\right)_{j \in \mathbb{N}} \subset(0,1)$ and $\mathbf{v}_{j}:=h_{j}^{-1}\left(\mathbf{W}_{*} x+\mathbf{W}_{*}^{2} x\right)$ a direct computation shows that

$$
\mathcal{F}_{h_{j}}^{I}\left(\mathbf{v}_{j}\right)=-h_{j}^{-1} \mathcal{L}\left(\mathbf{W}_{*}^{2} x\right) \rightarrow-\infty
$$

as $j \rightarrow+\infty$, as claimed.

For general $\Omega$, we notice that if the body is subject to a uniform boundary compressive force field then the above situation occurs. Indeed, if $\mathbf{n}$ denotes the outer unit normal vector to $\partial \Omega$, and we choose $\mathbf{g}=\lambda \mathbf{n}$ with $\lambda<0$ and $\mathbf{f} \equiv 0$, then

$$
\int_{\partial \Omega} \mathbf{g} \cdot \mathbf{W}^{2} x d \mathcal{H}^{2}(x)=\lambda\left(\operatorname{Tr} \mathbf{W}^{2}\right)|\Omega|>0 \quad \forall \mathbf{W} \in \mathbb{R}_{\text {skew }}^{3 \times 3}, \mathbf{W} \neq 0
$$

and by choosing $\mathbf{W} \in \mathbb{R}_{\text {skew }}^{3 \times 3}, \mathbf{W} \neq 0$ and $\mathbf{v}_{j}:=h_{j}^{-1}\left(\mathbf{W} x+\mathbf{W}^{2} x\right)$, we get

$$
\mathcal{F}_{h_{j}}^{I}\left(\mathbf{v}_{j}\right)=\mathcal{L}\left(\mathbf{v}_{j}\right)=-\frac{\lambda}{h_{j}} \int_{\partial \Omega} \mathbf{W}^{2} x \cdot \mathbf{n} d \mathcal{H}^{2}(x)=-\frac{\lambda}{h_{j}}\left(\operatorname{Tr} \mathbf{W}^{2}\right)|\Omega| \rightarrow-\infty
$$

as $j \rightarrow+\infty$, as before. On the other hand, if $\lambda>0$ we have a dilation effect on the body and $(\mathcal{L} 2)$ is satisfied.

Remark 2.8. Let us consider external forces of the following form. Given $p$ such that $(\mathcal{W} 4)$ holds, let $\mathbf{f}=\nabla \phi$, where $\phi \in W_{0}^{1, r}(\Omega), r=\frac{3 p}{4 p-3}$, and let $\mathbf{g}=\lambda \mathbf{n}$, where $\lambda \in \mathbb{R}$ and $\mathbf{n}$ is the unit exterior normal vector to $\partial \Omega$, with $\int_{\Omega} \phi(x) \mathrm{d} x<\lambda|\Omega|$. It is readily seen that in this case $(\mathcal{L} \mathbf{1})$ and $(\mathcal{L} 2)$ are satisfied. Moreover, by the divergence theorem, $\mathcal{L}(\mathbf{v})=0$ for every divergence-free vector field $\mathbf{v} \in H^{1}\left(\Omega, \mathbb{R}^{3}\right)$. Therefore, under the assumptions of Theorem 2.1, from the definition of $\mathcal{E}^{I}$ and from the estimate (3.5) below we deduce that $\operatorname{argmin}_{W^{1, p}\left(\Omega, \mathbb{R}^{3}\right)} \mathcal{E}^{I}$ coincides with the set of rigid displacements of $\Omega$ (i.e., displacements fields with vanishing infinitesimal strain tensor). From Theorem 2.1, we deduce in this case that the minimal value of both $\mathcal{E}^{I}$ and $\mathcal{F}^{I}$ is 0 . 


\section{Notation and preliminary results}

Through the paper, $\mathbb{R}^{3 \times 3}$ will denote the set of $3 \times 3$ real matrices. $\mathbb{R}_{\text {sym }}^{3 \times 3}$ and $\mathbb{R}_{\text {skew }}^{3 \times 3}$ denote, respectively, the sets of symmetric and skew-symmetric matrices and for every $\mathbf{B} \in \mathbb{R}^{3 \times 3}$ we define sym $\mathbf{B}:=\frac{1}{2}\left(\mathbf{B}+\mathbf{B}^{T}\right)$ and skew $\mathbf{B}:=\frac{1}{2}\left(\mathbf{B}-\mathbf{B}^{T}\right)$. Moreover, we set

$$
\mathbb{K}:=\{\tau(\mathbf{R}-\mathbf{I}): \tau \geq 0, \mathbf{R} \in S O(3)\} .
$$

Given $\mathbf{a}, \mathbf{b} \in \mathbb{R}^{3}$, with $\mathbf{a} \wedge \mathbf{b}$ we denote the cross product. A Sobolev vector field $\mathbf{w} \in W^{1,1}\left(\Omega, \mathbb{R}^{3}\right)$ is said to be an infinitesimal rigid displacement if $\mathbb{E}(\mathbf{v}):=\operatorname{sym} \nabla \mathbf{v}=0$ a.e. in $\Omega$, which is the case iff there exist $\mathbf{a}, \mathbf{b} \in \mathbb{R}^{3}$ such that $\mathbf{v}(x)=\mathbf{a} \wedge x+\mathbf{b}$ for every $x \in \Omega$. By $H_{\text {div }}^{1}\left(\Omega, \mathbb{R}^{3}\right)$, we denote the space of divergence-free $H^{1}\left(\Omega, \mathbb{R}^{3}\right)$ vector fields. The codomain of functions of $L^{r}(\Omega)$ or $W^{1, r}(\Omega)$ shall be $\mathbb{R}, \mathbb{R}^{3}$ or $\mathbb{R}^{3 \times 3}$ and we shall often omit it from the notation. Bold letters will be used for vector fields.

\section{Euler-Rodrigues formula}

For every $\mathbf{R} \in S O(3)$, there exist $\vartheta \in \mathbb{R}$ and $\mathbf{W} \in \mathbb{R}_{\text {skew }}^{3 \times 3}$, such that $|\mathbf{W}|^{2}=2$ and such that $\exp (\vartheta \mathbf{W})=\mathbf{R}$. By taking into account that $\mathbf{W}^{3}=-\mathbf{W}$, the exponential matrix series $\exp (\vartheta \mathbf{W})=\sum_{k=0}^{\infty} \vartheta^{k} \mathbf{W}^{k} / k$ ! yields the Euler-Rodrigues formula:

$$
\exp (\vartheta \mathbf{W})=\mathbf{R}=\mathbf{I}+\sin \vartheta \mathbf{W}+(1-\cos \vartheta) \mathbf{W}^{2} .
$$

We also recall that if $\mathbf{W} \in \mathbb{R}_{\text {skew }}^{3 \times 3}$ and $|\mathbf{W}|^{2}=2$ then $\left|\mathbf{W}^{2}\right|^{2}=2$.

\section{Properties of $\mathcal{W}$}

Let assumptions $(\mathcal{W} \mathbf{0}),(\mathcal{W} \mathbf{1}),(\mathcal{W} \mathbf{2}),(\mathcal{W} \mathbf{3})$ and $(\mathcal{W} \mathbf{4})$ hold. We recall that $\mathcal{W}$ is defined by $\mathcal{W}(x, \mathbf{F}):=$ $\mathcal{W}^{I}\left(x,(\operatorname{det} \mathbf{F})^{-1 / 3} \mathbf{F}\right)$, thus $\mathcal{W}^{I} \geq \mathcal{W}$. It is clear that $\mathcal{W}$ itself satisfies $(\mathcal{W} \mathbf{1})$ and $(\mathcal{W} \mathbf{2})$, so that by $(\mathcal{W} \mathbf{3})$, since $\mathcal{W} \geq 0$, we deduce

$$
\mathcal{W}(x, \mathbf{R})=0, D \mathcal{W}(x, \mathbf{R})=0 \quad \forall \mathbf{R} \in S O(3), \quad \text { for a.e. } x \in \Omega .
$$

Due to frame indifference, there exists a function $\mathcal{V}$ such that

$$
\mathcal{W}(x, \mathbf{F})=\mathcal{V}\left(x, \frac{1}{2}\left(\mathbf{F}^{T} \mathbf{F}-\mathbf{I}\right)\right), \quad \forall \mathbf{F} \in \mathbb{R}^{3 \times 3}, \text { for a.e. } x \in \Omega .
$$

Given $\mathbf{B} \in \mathbb{R}^{3 \times 3}, h>0$, we have $\mathcal{W}(x, \mathbf{I}+h \mathbf{B})=\mathcal{V}\left(x, h \operatorname{sym} \mathbf{B}+\frac{1}{2} h^{2} \mathbf{B}^{T} \mathbf{B}\right)$. By (3.3) and by ( $\left.\mathcal{W} \mathbf{3}\right)$, we get for a.e. $x \in \Omega$

$$
\lim _{h \rightarrow 0} h^{-2} \mathcal{W}(x, \mathbf{I}+h \mathbf{B})=\frac{1}{2} \operatorname{sym} \mathbf{B} D^{2} \mathcal{V}(x, \mathbf{0}) \operatorname{sym} \mathbf{B}=\frac{1}{2} \mathbf{B}^{T} D^{2} \mathcal{W}(x, \mathbf{I}) \mathbf{B},
$$

hence $(\mathcal{W} 4)$ and (2.3) imply that for a.e. $x \in \Omega$, as soon as $\operatorname{Tr} \mathbf{B}=0$,

$$
\begin{aligned}
\frac{1}{2} \mathbf{B}^{T} D^{2} \mathcal{W}(x, \mathbf{I}) \mathbf{B} & =\lim _{h \rightarrow 0} h^{-2} \mathcal{W}(x, \mathbf{I}+h \mathbf{B}+o(h))=\lim _{h \rightarrow 0} h^{-2} \mathcal{W}(x, \exp (h \mathbf{B})) \\
& =\lim _{h \rightarrow 0} h^{-2} \mathcal{W}^{I}(x, \exp (h \mathbf{B})) \geq \limsup _{h \rightarrow 0} C h^{-2} d^{2}(\exp (h \mathbf{B}), S O(3)) \\
& =\limsup _{h \rightarrow 0} C h^{-2}\left|\sqrt{\exp (h \mathbf{B})^{T} \exp (h \mathbf{B})}-\mathbf{I}\right|^{2} \\
& =\limsup _{h \rightarrow 0} C h^{-2}|\exp (h \operatorname{sym} \mathbf{B})-\mathbf{I}|^{2}=C|\operatorname{sym} \mathbf{B}|^{2}
\end{aligned}
$$


Moreover, by expressing the remainder of Taylor's expansion in terms of the $x$-independent modulus of continuity $\omega: \mathbb{R}_{+} \rightarrow \mathbb{R}$ of $D^{2} \mathcal{W}(x, \cdot)$ on the set $\mathcal{U}$ ( $\omega$ is an increasing function such that $\lim _{t \rightarrow 0^{+}} \omega(t)=0$ ) from $(\mathcal{W} 3)$, we have

$$
\left|\mathcal{W}(x, \mathbf{I}+h \mathbf{B})-\frac{h^{2}}{2} \operatorname{sym} \mathbf{B} D^{2} \mathcal{W}(x, \mathbf{I}) \operatorname{sym} \mathbf{B}\right| \leq h^{2} \omega(h|\mathbf{B}|)|\mathbf{B}|^{2}
$$

for any small enough $h$ (such that $h \mathbf{B} \in \mathcal{U}$ ), where $\omega: \mathbb{R}_{+} \rightarrow \mathbb{R}$ is such that $\lim _{t \rightarrow 0^{+}} \omega(t)=0$. Similarly, $\mathcal{V}(x, \cdot)$ is $C^{2}$ in a neighbor of the origin in $\mathbb{R}^{3 \times 3}$, with an $x$-independent modulus of continuity $\eta: \mathbb{R}_{+} \rightarrow \mathbb{R}$, which is increasing and such that $\lim _{t \rightarrow 0^{+}} \eta(t)=0$, and we have

$$
\left|\mathcal{V}(x, h \mathbf{B})-\frac{h^{2}}{2} \operatorname{sym} \mathbf{B} D^{2} \mathcal{V}(x, \mathbf{0}) \operatorname{sym} \mathbf{B}\right| \leq h^{2} \eta(h|\mathbf{B}|)|\mathbf{B}|^{2}
$$

for any small enough $h$.

\section{Sobolev-Poincaré inequality}

Here and for the rest of this section, $\Omega$ is a bounded connected set with Lipschitz boundary. Let $p \in(1,2]$. By Sobolev embedding, Sobolev trace embedding and by the Poincaré inequality for null-mean functions we have for any $\mathbf{v} \in W^{1, p}\left(\Omega, \mathbb{R}^{3}\right)$

$$
\|\mathbf{v}-\overline{\mathbf{v}}\|_{L^{\frac{3 p}{3-p}}\left(\Omega, \mathbb{R}^{3}\right)}+\|\mathbf{v}-\overline{\mathbf{v}}\|_{L^{\frac{2 p}{3-p}}\left(\partial \Omega, \mathbb{R}^{3}\right)} \leq K_{F}\|\nabla \mathbf{v}\|_{L^{p}\left(\Omega, \mathbb{R}^{3 \times 3}\right)},
$$

where $K_{F}$ is a constant only depending on $\Omega, p$ and $\overline{\mathbf{v}}:=\frac{1}{|\Omega|} \int_{\Omega} \mathbf{v} \mathrm{d} x$.

\section{Projection on rigid motions}

Let $p \in(1,2]$ and let

$$
\mathcal{R}:=\left\{\mathbf{v} \in W^{1,1}\left(\Omega, \mathbb{R}^{3}\right): \mathbb{E}(\mathbf{v})=\mathbf{0}\right\}
$$

denote the space spanned by the set of the infinitesimal rigid displacements. We denote by $\mathbb{P} \mathbf{v}$ the unique projection of $\mathbf{v} \in W^{1, p}\left(\Omega, \mathbb{R}^{3}\right)$ onto $\mathcal{R}$.

\section{Korn inequality}

Let $p \in(1,2]$. For any $\mathbf{v} \in W^{1, p}\left(\Omega, \mathbb{R}^{3}\right)$, there is a unique couple $\mathbf{W}_{\mathbf{v}} \in \mathbb{R}_{\text {skew }}^{3 \times 3}, \mathbf{a}_{\mathbf{v}} \in \mathbb{R}^{3}$ such that

$$
\|\mathbf{v}-\mathbb{P} \mathbf{v}\|_{L^{p}(\Omega)}=\min \left\{\|\mathbf{v}-(\mathbf{W} x+\mathbf{a})\|_{L^{p}(\Omega)}: \mathbf{W} \in \mathbb{R}_{\text {skew }}^{3 \times 3}, \mathbf{a} \in \mathbb{R}^{3}\right\}=\left\|\mathbf{v}-\left(\mathbf{W}_{\mathbf{v}} x+\mathbf{a}_{\mathbf{v}}\right)\right\|_{L^{p}(\Omega)}
$$

and Korn inequality, see for instance [27], entails the existence of a constant $Q_{K}=Q_{K}(\Omega, p)$ such that

$$
\left\|\nabla \mathbf{v}-\mathbf{W}_{\mathbf{v}}\right\|_{L^{p}(\Omega)} \leq Q_{K}\|\mathbb{E}(\mathbf{v})\|_{L^{p}(\Omega)} .
$$

Moreover, by combining the latter with Sobolev and trace inequalities, we obtain the existence of a further constant $C_{K}=C_{K}(\Omega, p)$ such that for all $\mathbf{v} \in W^{1, p}\left(\Omega, \mathbb{R}^{3}\right)$

$$
\|\mathbf{v}-\mathbb{P} \mathbf{v}\|_{L^{\frac{3 p}{3-p}}\left(\Omega, \mathbb{R}^{3}\right)}+\|\mathbf{v}-\mathbb{P} \mathbf{v}\|_{L^{\frac{2 p}{3-p}}\left(\partial \Omega, \mathbb{R}^{3}\right)} \leq C_{K}\|\mathbb{E}(\mathbf{v})\|_{L^{p}\left(\Omega, \mathbb{R}^{3 \times 3}\right)} .
$$




\section{Basic estimate on external forces}

As a consequence of $(3.10)$, if $(\mathcal{L} \mathbf{1})$ holds true we obtain the following estimate for functional $\mathcal{L}$ : for any $\mathbf{v} \in W^{1, p}\left(\Omega, \mathbb{R}^{3}\right)$, there holds

$$
\begin{aligned}
|\mathcal{L}(\mathbf{v})| & =|\mathcal{L}(\mathbf{v}-\mathbb{P} \mathbf{v})| \\
& \leq\|\mathbf{f}\|_{L^{\frac{3 p}{4 p-3}}\left(\Omega, \mathbb{R}^{3}\right)}\|\mathbf{v}-\mathbb{P} \mathbf{v}\|_{L^{\frac{3 p}{3-p}}\left(\Omega, \mathbb{R}^{3}\right)}+\|\mathbf{g}\|_{L^{\frac{2 p}{3 p-3}}\left(\partial \Omega, \mathbb{R}^{3}\right)}\|\mathbf{v}-\mathbb{P} \mathbf{v}\|_{L^{\frac{2 p}{3-p}}\left(\partial \Omega, \mathbb{R}^{3}\right)} \\
& \leq C_{\mathcal{L}}\|\mathbb{E}(\mathbf{v})\|_{L^{p}\left(\Omega, \mathbb{R}^{3 \times 3}\right)},
\end{aligned}
$$

where $C_{\mathcal{L}}:=C_{K}\left(\|\mathbf{f}\|_{L^{\frac{3 p}{4 p-3}}\left(\Omega, \mathbb{R}^{3}\right)}+\|\mathbf{g}\|_{L^{\frac{2 p}{3 p-3}}\left(\partial \Omega, \mathbb{R}^{3}\right)}\right)$ and $C_{K}$ is the constant in (3.10).

\section{Rigidity inequality}

We recall the rigidity inequality by Friesecke, James and Müller [12], in its version from [13], [2]. Let $g_{p}$ the function defined in (2.2). There exists a constant $C_{p}=C_{p}(\Omega)>0$ such that for every $\mathbf{y} \in W^{1, p}\left(\Omega, \mathbb{R}^{3}\right)$ there exists a constant $\mathbf{R} \in S O(3)$ such that we have

$$
\int_{\Omega} g_{p}(|\nabla \mathbf{y}-\mathbf{R}|) \mathrm{d} x \leq C_{p} \int_{\Omega} g_{p}(d(\nabla \mathbf{y}, S O(3))) \mathrm{d} x .
$$

We close this section with a result about convergence of infinitesimal strain tensors.

Lemma 3.1. Let $p \in(1,2]$. Let $\left(\boldsymbol{w}_{n}\right)_{n \in \mathbb{N}} \subset W^{1, p}\left(\Omega, \mathbb{R}^{3}\right)$ be a sequence such that $\mathbb{E}\left(\boldsymbol{w}_{n}\right) \rightarrow \boldsymbol{T}$ weakly in $L^{p}\left(\Omega, \mathbb{R}^{3 \times 3}\right)$ as $n \rightarrow+\infty$. Then, there exists $\boldsymbol{w} \in W^{1, p}\left(\Omega, \mathbb{R}^{3}\right)$ such that $\boldsymbol{T}=\mathbb{E}(\boldsymbol{w})$. If in addition we assume that $\nabla \boldsymbol{w}_{n} \rightarrow \boldsymbol{G}$ weakly in $L^{p}\left(\Omega ; \mathbb{R}^{3 \times 3}\right)$, then there exists a constant matrix $\boldsymbol{W} \in \mathbb{R}_{\text {skew }}^{3 \times 3}$ such that $\nabla \boldsymbol{w}=\boldsymbol{G}-\boldsymbol{W}$.

Proof. The proof is given in $[24$, Lemma 3.2] for the case $p=2$. Its extension to $p \in(1,2)$ is straightforward.

\section{Compactness}

We prove uniform $L^{p}\left(\Omega, \mathbb{R}^{3 \times 3}\right)$ bounds for $\mathbb{E}\left(\mathbf{v}_{j}\right)$ on almost minimizing sequences $\left(\mathbf{v}_{j}\right)_{j \in \mathbb{N}}$ of $\mathcal{F}_{h_{j}}^{I}\left(\mathbf{v}_{j}\right)$ as $h_{j} \rightarrow 0$. We start by showing that functionals $\mathcal{F}_{h}^{I}$ are uniformly bounded from below.

Lemma 4.1. (Boundedness from below) Assume $(2.1),(\mathcal{W} \mathbf{0}),(\mathcal{W} \mathbf{1}),(\mathcal{W} \mathbf{2}),(\mathcal{W} \mathbf{3}),(\mathcal{W} \mathbf{4}),(\mathcal{L} 1)$ and $(\mathcal{L} 2)$. There exists a constant $C>0$ (only depending on $\Omega, p, \boldsymbol{f}, \boldsymbol{g}$ ) such that $\mathcal{F}_{h}^{I}(\mathbf{v}) \geq-C$ for any $h \in(0,1)$ and any $\mathbf{v} \in W^{1, p}\left(\Omega, \mathbb{R}^{3}\right)$.

Proof. Let $\mathbf{v} \in W^{1, p}\left(\Omega, \mathbb{R}^{3}\right)$ and let $h \in(0,1)$. Let $\mathbf{y}:=\boldsymbol{i}+h \mathbf{v}$, where $\boldsymbol{i}$ denotes the identity map on $\mathbb{R}^{3}$, and let $\mathbf{R} \in S O(3)$ be a constant matrix such that (3.12) holds. Let $S:=\{x \in \Omega:|\nabla \mathbf{y}(x)-\mathbf{R}| \leq 1\}$. By taking advantage of assumption $\mathcal{W} 4$ and of the linearity of $\mathcal{L}$, since $g_{p}(t)=t^{2}$ for $0 \leq t \leq 1$ and $g_{p}(t) \geq t^{p}$ for $t \geq 1$, we get

$$
\begin{aligned}
\mathcal{F}_{h}^{I}(\mathbf{v}) & \geq \frac{c}{h^{2}} \int_{\Omega} g_{p}(|\nabla \mathbf{y}-\mathbf{R}|) \mathrm{d} x-\frac{1}{h} \mathcal{L}(\mathbf{y}-\boldsymbol{i}) \\
& \geq \frac{c}{h^{2}} \int_{S}|\nabla \mathbf{y}-\mathbf{R}|^{2} \mathrm{~d} x+\frac{c}{h^{2}} \int_{\Omega \backslash S}|\nabla \mathbf{y}-\mathbf{R}|^{p} \mathrm{~d} x-\frac{1}{h} \mathcal{L}(\mathbf{y}-\boldsymbol{i}),
\end{aligned}
$$


where $c>0$ is a constant only depending on $p$ and $\Omega$. By the Sobolev-Poincaré inequality (3.8), letting $\mathbf{u}(x):=\mathbf{R} x$ and letting $\mathbf{m}$ denote the mean value of $\mathbf{y}-\mathbf{u}$ on $\Omega$, we have

$$
\|\mathbf{y}-\mathbf{u}-\mathbf{m}\|_{L^{\frac{3 p}{3-p}}\left(\Omega, \mathbb{R}^{3}\right)}+\|\mathbf{y}-\mathbf{u}-\mathbf{m}\|_{L^{\frac{2 p}{3-p}}\left(\partial \Omega, \mathbb{R}^{3}\right)} \leq K_{F}\|\nabla \mathbf{y}-\mathbf{R}\|_{L^{p}\left(\Omega, \mathbb{R}^{3 \times 3}\right)} .
$$

By the Euler-Rodrigues formula (3.2), we represent $\mathbf{R}$ as $\mathbf{R}=\mathbf{I}+\sin \theta \mathbf{W}+(1-\cos \theta) \mathbf{W}^{2}$ for a suitable skew-symmetric matrix $\mathbf{W}$ and some $\theta \in(-\pi, \pi]$. Then, we notice that $(\mathcal{L} \mathbf{1})$ and $(\mathcal{L} 2)$ entail $\mathcal{L}(\mathbf{u}-\boldsymbol{i}) \leq 0$ so that $\mathcal{L}(\mathbf{y}-\boldsymbol{i}) \leq \mathcal{L}(\mathbf{y}-\mathbf{u}-\mathbf{m})$. As a consequence, by means of the Hölder inequality and of (4.2) we get

$$
\begin{aligned}
& \mathcal{L}(\mathbf{y}-\boldsymbol{i}) \leq \mathcal{L}(\mathbf{y}-\mathbf{u}-\mathbf{m}) \\
& \quad \leq\|\mathbf{f}\|_{L^{\frac{3 p}{4 p-3}}\left(\Omega, \mathbb{R}^{3}\right)}\|\mathbf{y}-\mathbf{u}-\mathbf{m}\|_{L^{\frac{3 p}{3-p}}\left(\Omega, \mathbb{R}^{3}\right)}+\|\mathbf{g}\|_{L^{\frac{2 p}{3 p-3}}\left(\partial \Omega, \mathbb{R}^{3}\right)}\|\mathbf{y}-\mathbf{u}-\mathbf{m}\|_{L^{\frac{2 p}{3-p}}\left(\partial \Omega, \mathbb{R}^{3}\right)} \\
& \quad \leq C_{\mathbf{f}, \mathbf{g}}\|\nabla \mathbf{y}-\mathbf{R}\|_{L^{p}\left(\Omega, \mathbb{R}^{3 \times 3}\right)} \leq C_{\mathbf{f}, \mathbf{g}}\|\nabla \mathbf{y}-\mathbf{R}\|_{L^{p}\left(\Omega \backslash S, \mathbb{R}^{3 \times 3}\right)}+C_{\mathbf{f}, \mathbf{g}}|\Omega|^{\frac{2-p}{2 p}}\|\nabla \mathbf{y}-\mathbf{R}\|_{L^{2}\left(S, \mathbb{R}^{3 \times 3}\right)}
\end{aligned}
$$

where $C_{\mathbf{f}, \mathbf{g}}:=K_{F}\left(\|\mathbf{f}\|_{L^{\frac{3 p}{4 p-3}}\left(\Omega, \mathbb{R}^{3}\right)}+\|\mathbf{g}\|_{L^{\frac{p}{p-1}}\left(\partial \Omega, \mathbb{R}^{3}\right)}\right)$, and then by Young inequality we obtain

$$
\begin{aligned}
\mathcal{L}(\mathbf{y}-\boldsymbol{i}) \leq & \frac{p-1}{p} C_{\mathbf{f}, \mathbf{g}}^{\frac{p}{p-1}}\left(\frac{2 h}{c p}\right)^{\frac{1}{p-1}}+\frac{c}{2 h}\|\nabla \mathbf{y}-\mathbf{R}\|_{L^{p}\left(\Omega \backslash S, \mathbb{R}^{3 \times 3}\right)}^{p} \\
& +\frac{h}{2 c} C_{\mathbf{f}, \mathbf{g}}^{2}|\Omega|^{\frac{2-p}{p}}+\frac{c}{2 h}\|\nabla \mathbf{y}-\mathbf{R}\|_{L^{2}\left(S, \mathbb{R}^{3 \times 3}\right)}^{2},
\end{aligned}
$$

where $c$ is the constant appearing in (4.1). By joining together (4.1) and (4.3), we get

$$
\begin{aligned}
\mathcal{F}_{h}^{I}(\mathbf{v}) \geq & \frac{c}{2 h^{2}}\|\nabla \mathbf{y}-\mathbf{R}\|_{L^{p}\left(\Omega \backslash S, \mathbb{R}^{3 \times 3}\right)}^{p}+\frac{c}{2 h^{2}}\|\nabla \mathbf{y}-\mathbf{R}\|_{L^{2}\left(S, \mathbb{R}^{3 \times 3}\right)}^{2} \\
& -C_{\mathbf{f}, \mathbf{g}}^{\frac{p}{p-1}}\left(\frac{2}{c p}\right)^{\frac{1}{p-1}} h^{\frac{2-p}{p-1}}-\frac{1}{2 c} C_{\mathbf{f}, \mathbf{g}}^{2}|\Omega|^{\frac{2-p}{p}} \geq-C_{\mathbf{f}, \mathbf{g}}^{\frac{p}{p-1}}\left(\frac{2}{c p}\right)^{\frac{1}{p-1}}-\frac{1}{2 c} C_{\mathbf{f}, \mathbf{g}}^{2}|\Omega|^{\frac{2-p}{p}}
\end{aligned}
$$

as desired.

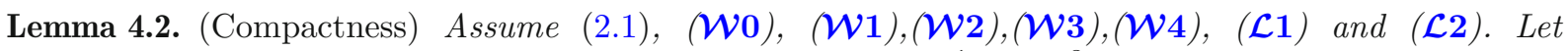
$\left(h_{j}\right)_{j \subset \mathbb{N}} \subset(0,1)$ be a vanishing a sequence and let $\left(\mathbf{v}_{j}\right)_{j \in \mathbb{N}} \subset W^{1, p}\left(\Omega, \mathbb{R}^{3}\right)$ be a sequence such that

$$
\lim _{j \rightarrow+\infty}\left(\mathcal{F}_{h_{j}}^{I}\left(\mathbf{v}_{j}\right)-\inf _{W^{1, p}(\Omega)} \mathcal{F}_{h_{j}}^{I}\right)=0 .
$$

Then, there exists $M>0$ such that $\left\|\mathbb{E}\left(\mathbf{v}_{j}\right)\right\|_{L^{p}(\Omega)} \leq M$ for any $j \in \mathbb{N}$.

Proof. The argument extends the one of [24, Lemma 3.6] to the weaker coercivity condition ( $\mathcal{W} 4)$.

For any $j \in \mathbb{N}$, by Lemma 4.1 there holds

$$
-\infty<\inf _{W^{1, p}(\Omega)} \mathcal{F}_{h_{j}}^{I} \leq \mathcal{F}_{h_{j}}^{I}(\mathbf{0})=0,
$$

therefore by considering (4.4) it is not restrictive to assume that $\mathcal{F}_{h_{j}}^{I}\left(\mathbf{v}_{j}\right) \leq 1$ for any $j \in \mathbb{N}$. We assume by contradiction that the sequence $\left(t_{j}\right)_{j \in \mathbb{N}}$, defined as $t_{j}:=\left\|\mathbb{E}\left(\mathbf{v}_{j}\right)\right\|_{L^{p}(\Omega)}$, is unbounded, so that up to extraction of a not relabeled subsequence we have $t_{j} \rightarrow+\infty$ as $j \rightarrow+\infty$ and moreover $t_{j} h_{j}$ converge to a limit as $j \rightarrow+\infty$. We let $\mathbf{w}_{j}=\mathbf{v}_{j} / t_{j}$, so that $\left\|\mathbb{E}\left(\mathbf{w}_{j}\right)\right\|_{L^{p}(\Omega)}=1$ for any $j \in \mathbb{N}$, and along a not relabeled subsequence we have $\mathbb{E}\left(\mathbf{w}_{j}\right) \rightarrow \mathbb{E}(\mathbf{w})$ weakly in $L^{p}(\Omega)$ for some $\mathbf{w} \in W^{1, p}(\Omega)$, thanks to Lemma 3.1. By defining $\mathbf{y}_{j}:=\boldsymbol{i}+h_{j} \mathbf{v}_{j}$, we let $\mathbf{R}_{j}$ be the corresponding constant rotation matrix such that (3.12) holds, so that by $(\mathcal{W} 4)$, since $\mathcal{F}_{h_{j}}^{I}\left(\mathbf{v}_{j}\right) \leq 1$, we get

$$
\int_{\Omega} g_{p}\left(\left|\nabla \mathbf{y}_{j}-\mathbf{R}_{j}\right|\right) \mathrm{d} x \leq \int_{\Omega} g_{p}\left(d\left(\nabla \mathbf{y}_{j}, S O(3)\right)\right) \mathrm{d} x \leq \int_{\Omega} \mathcal{W}\left(x, \mathbf{I}+h_{j} \nabla \mathbf{v}_{j}\right) \mathrm{d} x \leq h_{j}^{2}+h_{j}^{2} \mathcal{L}\left(\mathbf{v}_{j}\right) .
$$


By inserting (3.11)

$$
\int_{\Omega} g_{p}\left(\left|\nabla \mathbf{y}_{j}-\mathbf{R}_{j}\right|\right) \mathrm{d} x \leq h_{j}^{2}+h_{j}^{2} \mathcal{L}\left(\mathbf{v}_{j}\right) \leq h_{j}^{2}+C_{\mathcal{L}} h_{j}^{2}\left\|\mathbb{E}\left(\mathbf{v}_{j}\right)\right\|_{L^{p}(\Omega)}=h_{j}^{2}\left(1+C_{\mathcal{L}} t_{j}\right),
$$

that is,

$$
\int_{\Omega} g_{p}\left(\left|\mathbf{I}-\mathbf{R}_{j}+t_{j} h_{j} \nabla \mathbf{w}_{j}\right|\right) \mathrm{d} x \leq h_{j}^{2}\left(1+C_{\mathcal{L}} t_{j}\right) .
$$

We claim that $\nabla \mathbf{w}$ is the sum of a skew-symmetric matrix and an element of $\mathbb{K}$, where $\mathbb{K}$ is defined by (3.1), and that $\mathbb{E}\left(\mathbf{w}_{j}\right) \rightarrow \mathbb{E}(\mathbf{w})$ in $L^{p}(\Omega)$, up to extraction of a further not relabeled subsequence. We shall prove the claim by separately treating the following three possible cases: $h_{j} t_{j} \rightarrow \lambda \in(0,+\infty), h_{j} t_{j} \rightarrow 0$ and $h_{j} t_{j} \rightarrow+\infty$ as $j \rightarrow+\infty$.

Case $1 h_{j} t_{j} \rightarrow \lambda$ as $j \rightarrow+\infty$ for some $\lambda>0$. It is easy to check that

$$
2 g_{p}(a x) \geq\left(a^{2} \wedge a^{p}\right) g_{p}(x) \quad \text { for any } x \geq 0 \text { and any } a \geq 0,
$$

so that (4.6) implies

$$
\begin{aligned}
\frac{1}{2}\left(\left(h_{j}^{2} t_{j}^{2}\right) \wedge\left(h_{j}^{p} t_{j}^{p}\right)\right) \int_{\Omega} g_{p}\left(\left|\frac{\mathbf{I}-\mathbf{R}_{j}}{h_{j} t_{j}}+\nabla \mathbf{w}_{j}\right|\right) \mathrm{d} x & \leq \int_{\Omega} g_{p}\left(\left|\mathbf{I}-\mathbf{R}_{j}+h_{j} t_{j} \nabla \mathbf{w}_{j}\right|\right) \mathrm{d} x \\
& \leq h_{j}^{2}\left(1+C_{\mathcal{L}} t_{j}\right),
\end{aligned}
$$

therefore

$$
\lim _{j \rightarrow+\infty} \int_{\Omega} g_{p}\left(\left|\frac{\mathbf{I}-\mathbf{R}_{j}}{h_{j} t_{j}}+\nabla \mathbf{w}_{j}\right|\right) \mathrm{d} x=0 .
$$

We define

$$
A_{j}:=\left\{x \in \Omega:\left|\mathbf{I}-\mathbf{R}_{j}+t_{j} h_{j} \nabla \mathbf{w}_{j}(x)\right| \leq t_{j} h_{j}\right\}
$$

Since $g_{p}(t)=t^{2}$ for $0 \leq t \leq 1$ and $g_{p}(t) \geq t^{p}$ for $t \geq 1$, taking advantage of (4.8) we get

$$
\lim _{j \rightarrow+\infty} \int_{\Omega} \chi_{A_{j}}\left|\frac{\mathbf{I}-\mathbf{R}_{j}}{h_{j} t_{j}}+\nabla \mathbf{w}_{j}\right|^{2} \mathrm{~d} x+\lim _{j \rightarrow+\infty} \int_{\Omega}\left(1-\chi_{A_{j}}\right)\left|\frac{\mathbf{I}-\mathbf{R}_{j}}{h_{j} t_{j}}+\nabla \mathbf{w}_{j}\right|^{p} \mathrm{~d} x=0
$$

so that both

$$
\left(1-\chi_{A_{j}}\right)\left(\frac{\mathbf{I}-\mathbf{R}_{j}}{h_{j} t_{j}}+\nabla \mathbf{w}_{j}\right) \quad \text { and } \quad \chi_{A_{j}}\left(\frac{\mathbf{I}-\mathbf{R}_{j}}{h_{j} t_{j}}+\nabla \mathbf{w}_{j}\right)
$$

go to zero in $L^{p}(\Omega)$ as $j \rightarrow+\infty$, since $p \in(1,2]$. As a consequence, we obtain the convergence to zero of $h_{j}^{-1} t_{j}^{-1}\left(\mathbf{I}-\mathbf{R}_{j}\right)+\nabla \mathbf{w}_{j}$ in $L^{p}(\Omega)$ as $j \rightarrow+\infty$. Therefore, $\nabla \mathbf{w}_{j}$ converge in $L^{p}(\Omega)$, up to subsequences, to $\lambda^{-1}(\mathbf{R}-\mathbf{I})$ for some suitable $\mathbf{R} \in S O(3)$ (thus $\mathbb{E}\left(\mathbf{w}_{j}\right)$ converge in $L^{p}(\Omega)$ to $\mathbb{E}(\mathbf{w})$ ) and Lemma 3.1 implies that $\nabla \mathbf{w}$ is the sum of a constant skew-symmetric matrix and an element of $\mathbb{K}$.

Case $2 h_{j} t_{j} \rightarrow 0$ as $j \rightarrow+\infty$. We assume w.l.o.g. that $t_{j} h_{j} \leq 1$ for any $j \in \mathbb{N}$. Writing $\mathbf{R}_{j}$ by means of the Euler-Rodrigues formula (3.2), from (4.6) we get

$$
\int_{\Omega} g_{p}\left(\left|h_{j} t_{j} \nabla \mathbf{w}_{j}-\sin \theta_{j} \mathbf{W}_{j}-(1-\cos \theta) \mathbf{W}_{j}^{2}\right|\right) \mathrm{d} x \leq h_{j}^{2}\left(1+C_{\mathcal{L}} t_{j}\right),
$$

where, for any $j \in \mathbb{N}, \theta_{j} \in(-\pi, \pi]$ and $\mathbf{W}_{j} \in \mathbb{R}^{3 \times 3}$ is skew-symmetric. Since $\mid$ sym $\mathbf{F}|\leq| \mathbf{F} \mid$ and $g_{p}$ is increasing, we deduce

$$
\int_{\Omega} g_{p}\left(\left|h_{j} t_{j} \mathbb{E}(\mathbf{w})-(1-\cos \theta) \mathbf{W}_{j}^{2}\right|\right) \mathrm{d} x \leq h_{j}^{2}\left(1+C_{\mathcal{L}} t_{j}\right) .
$$


Therefore, (4.7) implies (since $h_{j} t_{j} \leq 1$ )

$$
\int_{\Omega} g_{p}\left(\left|\mathbb{E}\left(\mathbf{w}_{j}\right)-\frac{\left(1-\cos \theta_{j}\right) \mathbf{W}_{j}^{2}}{h_{j} t_{j}}\right|\right) \mathrm{d} x \leq \frac{2+2 C_{\mathcal{L}} t_{j}}{t_{j}^{2}} .
$$

By taking advantage of the latter estimate, since $g_{p}$ is increasing and satisfies $g_{p}(x) \leq 2 x^{p}$ for any $x \geq 0$, we get

$$
\begin{aligned}
|\Omega| g_{p}\left(\left|\frac{\left(1-\cos \theta_{j}\right) \mathbf{W}_{j}^{2}}{h_{j} t_{j}}\right|\right) & =\int_{\Omega} g_{p}\left(\left|\frac{\left(1-\cos \theta_{j}\right) \mathbf{W}_{j}^{2}}{h_{j} t_{j}}\right|\right) \mathrm{d} x \\
& \leq \int_{\Omega} g_{p}\left(\left|\mathbb{E}\left(\mathbf{w}_{j}\right)\right|\right) \mathrm{d} x+\int_{\Omega} g_{p}\left(\left|\mathbb{E}\left(\mathbf{w}_{j}\right)-\frac{\left(1-\cos \theta_{j}\right) \mathbf{W}_{j}^{2}}{h_{j} t_{j}}\right|\right) \mathrm{d} x \\
& \leq 2 \int_{\Omega}\left|\mathbb{E}\left(\mathbf{w}_{j}\right)\right|^{p} \mathrm{~d} x+\int_{\Omega} g_{p}\left(\left|\mathbb{E}\left(\mathbf{w}_{j}\right)-\frac{\left(1-\cos \theta_{j}\right) \mathbf{W}_{j}^{2}}{h_{j} t_{j}}\right|\right) \mathrm{d} x \\
& \leq 2+\frac{2+2 C_{\mathcal{L}} t_{j}}{t_{j}^{2}} .
\end{aligned}
$$

Since $t_{j} \rightarrow+\infty$ as $j \rightarrow+\infty$, we obtain the existence of a positive constant $C_{*}$ (not depending on $j$ ) such that

$$
\frac{1-\cos \theta_{j}}{h_{j} t_{j}}=\frac{\sqrt{2}}{2}\left|\frac{\left(1-\cos \theta_{j}\right) \mathbf{W}_{j}^{2}}{h_{j} t_{j}}\right| \leq C_{*} .
$$

In particular, up to subsequences, we have

$$
\lim _{j \rightarrow+\infty} \frac{\left(1-\cos \theta_{j}\right) \mathbf{W}_{j}^{2}}{h_{j} t_{j}}=\mathbf{G}^{2}
$$

for some suitable constant skew-symmetric matrix $\mathbf{G}$, so that from (4.9) we deduce (since $g_{p}$ is continuous and increasing, and since from (2.2) it is possible to check that there exists a positive constant $c_{p}$ such that $g_{p}(a+b) \leq c_{p}\left(g_{p}(a)+g_{p}(b)\right)$ for any $a \geq 0$ and any $\left.b \geq 0\right)$

$$
\begin{aligned}
\limsup _{j \rightarrow+\infty} \int_{\Omega} g_{p}\left(\left|\mathbb{E}\left(\mathbf{w}_{j}\right)-\mathbf{G}^{2}\right|\right) \mathrm{d} x \leq & c_{p} \limsup _{j \rightarrow+\infty} \int_{\Omega} g_{p}\left(\left|\mathbb{E}\left(\mathbf{w}_{j}\right)-\frac{\left(1-\cos \theta_{j}\right) \mathbf{W}_{j}^{2}}{h_{j} t_{j}}\right|\right) \mathrm{d} x \\
& +c_{p} \limsup _{j \rightarrow+\infty} \int_{\Omega} g_{p}\left(\left|\frac{\left(1-\cos \theta_{j}\right) \mathbf{W}_{j}^{2}}{h_{j} t_{j}}-\mathbf{G}^{2}\right|\right) \mathrm{d} x=0 .
\end{aligned}
$$

By the same argument of Case 1 , we conclude that $\mathbb{E}\left(\mathbf{w}_{j}\right) \rightarrow \mathbf{G}^{2}$ in $L^{p}(\Omega)$ as $j \rightarrow+\infty$, hence $\mathbb{E}(\mathbf{w})=\mathbf{G}^{2}$, thus $\nabla \mathbf{w}=\operatorname{Skew}(\nabla \mathbf{w})+\mathbf{G}^{2}$. We deduce that the skew-symmetric part of $\nabla \mathbf{w}$ is a gradient field, hence a constant skew-symmetric matrix $\boldsymbol{\Lambda}$, and that $\nabla \mathbf{w}=\boldsymbol{\Lambda}+\mathbf{G}^{2}$.

By applying the Euler-Rodrigues formula (3.2), we deduce the existence of $\mu>0$, of $\mathbf{R} \in S O(3)$ and of $\mathbf{Q} \in \mathbb{R}_{\text {skew }}^{3 \times 3}$ such that $\nabla \mathbf{w}=\mu(\mathbf{R}-\mathbf{I})+\mathbf{Q}$, so that indeed $\nabla \mathbf{w}$ is the sum of an element of $\mathbb{K}$ and a constant skew-symmetric matrix.

Case $3 h_{j} t_{j} \rightarrow+\infty$ as $j \rightarrow+\infty$. We may assume in this case that $h_{j} t_{j} \geq 1$ for any $j \in \mathbb{N}$. By applying (4.6) and (4.7), we get

$$
\int_{\Omega} g_{p}\left(\left|\frac{\mathbf{I}-\mathbf{R}_{j}}{h_{j} t_{j}}+\nabla \mathbf{w}_{j}\right|\right) \mathrm{d} x \leq \frac{2 h_{j}^{2}\left(1+C_{\mathcal{L}} t_{j}\right)}{h_{j}^{p} t_{j}^{p}},
$$


where the right-hand side vanishes as $j \rightarrow+\infty$, and where $\frac{\mathbf{I}-\mathbf{R}_{j}}{h_{j} t_{j}}$ vanishes as well, since $\mathbf{R}_{j}-\mathbf{I}$ is bounded. By the same argument of Case 1 , we conclude that $\nabla \mathbf{w}_{j} \rightarrow \mathbf{0}$ in $L^{p}(\Omega)$ as $j \rightarrow+\infty$, thus $\mathbb{E}\left(\mathbf{w}_{j}\right) \rightarrow \mathbb{E}(\mathbf{w})=\mathbf{0}$ in $L^{p}(\Omega)$. By Lemma 3.1, we deduce that $\nabla \mathbf{w}$ is a constant skew-symmetric matrix. This ends the last of the three cases and proves the claim.

Let $\tilde{\mathbf{w}}_{j}:=\mathbf{w}_{j}-\mathbb{P} \mathbf{w}_{j}$ so that $\mathbb{E}\left(\tilde{\mathbf{w}}_{j}\right)=\mathbb{E}\left(\mathbf{w}_{j}\right)$ and $\mathcal{L}\left(\tilde{\mathbf{w}}_{j}\right)=\mathcal{L}\left(\mathbf{w}_{j}\right)$ by $(\mathcal{L} \mathbf{1})$. The claim we just proved implies $\mathbb{E}\left(\tilde{\mathbf{w}}_{j}\right) \rightarrow \mathbb{E}(\mathbf{w})$ in $L^{p}(\Omega)$ as $j \rightarrow+\infty$. Therefore, since (3.11) implies

$$
\left|\mathcal{L}\left(\tilde{\mathbf{w}}_{j}\right)-\mathcal{L}(\mathbf{w})\right|=\left|\mathcal{L}\left(\tilde{\mathbf{w}}_{j}-\mathbf{w}\right)\right| \leq C_{\mathcal{L}}\left\|\mathbb{E}\left(\tilde{\mathbf{w}}_{j}\right)-\mathbb{E}(\mathbf{w})\right\|_{L^{p}(\Omega)},
$$

we deduce $\mathcal{L}\left(\mathbf{w}_{j}\right)=\mathcal{L}\left(\tilde{\mathbf{w}}_{j}\right) \rightarrow \mathcal{L}(\mathbf{w})$ as $j \rightarrow+\infty$. As a consequence, thanks to (4.4) and (4.5) we infer that

$$
\begin{aligned}
\mathcal{L}(\mathbf{w}) & =\lim _{j \rightarrow+\infty} \mathcal{L}\left(\mathbf{w}_{j}\right)=\lim _{j \rightarrow+\infty} \frac{1}{t_{j}} \mathcal{L}\left(\mathbf{v}_{j}\right) \\
& =\lim _{j \rightarrow+\infty} \frac{1}{t_{j}}\left(\frac{1}{h_{j}^{2}} \int_{\Omega} \mathcal{W}^{I}\left(x, \mathbf{I}+h_{j} \nabla \mathbf{v}_{j}\right) \mathrm{d} x-\mathcal{F}_{h_{j}}^{I}\left(\mathbf{v}_{j}\right)\right) \geq 0 .
\end{aligned}
$$

Since we have already proven that $\nabla \mathbf{w}$ is the sum of a constant skew-symmetric matrix and an element of $\mathbb{K}$, we have $\mathbf{w}(\mathbf{x})=\tau(\mathbf{R}-\mathbf{I}) \mathbf{x}+\mathbf{A} \mathbf{x}+\mathbf{c}$ for suitable $\tau \geq 0, \mathbf{R} \in S O(3)$ such that $\mathbf{R} \neq \mathbf{I}, \mathbf{A} \in \mathbf{R}_{\text {skew }}^{3 \times 3}$ and $\mathbf{c} \in \mathbb{R}^{3}$. By (3.2) there exist $\vartheta \in(-\pi, \pi], \theta \neq 0$, and $\mathbf{W} \in \mathbb{R}_{\text {skew }}^{3 \times 3}, \mathbf{W} \neq \mathbf{0}$, such that $\mathbf{R}=\mathbf{I}+(1-\cos \vartheta) \mathbf{W}^{2}+(\sin \vartheta) \mathbf{W}$. Hence, $(\mathcal{L} 1)$ and $(4.10)$ yield

$$
\begin{aligned}
0 \leq \mathcal{L}(\mathbf{w})= & \tau \int_{\partial \Omega} \mathbf{g} \cdot(\mathbf{R}-\mathbf{I}) x d \mathcal{H}^{2}+\tau \int_{\Omega} \mathbf{f} \cdot(\mathbf{R}-\mathbf{I}) x \mathrm{~d} x \\
& =\tau(1-\cos \vartheta)\left(\int_{\partial \Omega} \mathbf{g} \cdot \mathbf{W}^{2} x d \mathcal{H}^{2}+\int_{\Omega} \mathbf{f} \cdot \mathbf{W}^{2} x \mathrm{~d} x\right) .
\end{aligned}
$$

By taking $(\mathcal{L} 2)$ into account, we conclude that $\tau=0$, so that $\nabla \mathbf{w}$ is a constant skew-symmetric matrix and then $\mathbb{E}(\mathbf{w})=\mathbf{0}$. But $\mathbb{E}\left(\mathbf{w}_{j}\right) \rightarrow \mathbb{E}(\mathbf{w})$ in $L^{p}(\Omega)$ and $\left\|\mathbb{E}\left(\mathbf{w}_{j}\right)\right\|_{L^{p}(\Omega)}=1$ imply $\|\mathbb{E}(\mathbf{w})\|_{L^{p}(\Omega)}=1$, a contradiction.

\section{Lower bound}

In this section, we prove the lower bound $\liminf \inf _{j \rightarrow+\infty} \mathcal{F}_{h_{j}}^{I}\left(\mathbf{v}_{j}\right) \geq \mathcal{F}^{I}(\mathbf{v})$ as $\mathbb{E}\left(\mathbf{v}_{j}\right) \rightarrow \mathbb{E}(\mathbf{v})$ weakly in $L^{p}\left(\Omega, \mathbb{R}^{3 \times 3}\right)$ and $h_{j} \rightarrow 0$. We start with two preliminary lemmas.

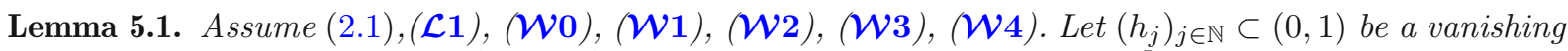
sequence and let $\left(\mathbf{v}_{j}\right)_{j \in \mathbb{N}} \subset W^{1, p}\left(\Omega, \mathbb{R}^{3}\right)$ be a sequence such that $\sup _{j \in \mathbb{N}} \mathcal{F}_{h_{j}}^{I}\left(\mathbf{v}_{j}\right)<+\infty$ and such that $\mathbb{E}\left(\mathbf{v}_{j}\right) \rightarrow \mathbb{E}(\mathbf{v})$ weakly in $L^{p}\left(\Omega, \mathbb{R}^{3 \times 3}\right)$ as $j \rightarrow \infty$ for some $\mathbf{v} \in W^{1, p}\left(\Omega, \mathbb{R}^{3}\right)$. Then, there exists a constant matrix $\boldsymbol{W} \in \mathbb{R}_{\text {skew }}^{3 \times 3}$ such that, up to subsequences, $\sqrt{h}, \nabla \mathbf{v}_{j} \rightarrow \boldsymbol{W}$ in $L^{p}\left(\Omega, \mathbb{R}^{3 \times 3}\right)$ as $j \rightarrow \infty$.

Proof. Through the proof, $C$ will always denote a generic positive constant only depending on $p, \Omega, \mathbf{f}$ and $\mathbf{g}$. By taking into account $(\mathcal{L} 1),(\mathcal{W} 4)$, and by applying $(3.12)$ to $\mathbf{y}_{j}:=\mathbf{i}+h_{j} \mathbf{v}_{j}$, we see that for any $j \in \mathbb{N}$ there exists a constant matrix $\mathbf{R}_{j} \in S O(3)$ such that

$$
h_{j}^{-2} \int_{\Omega} g_{p}\left(\left|\mathbf{I}+h_{j} \nabla \mathbf{v}_{j}-\mathbf{R}_{j}\right|\right) \mathrm{d} x-\mathcal{L}\left(\mathbf{v}_{j}-\mathbb{P} \mathbf{v}_{j}\right) \leq C .
$$


By (3.11) and by the boundedness of $\mathbb{E}\left(\mathbf{v}_{j}\right)$ in $L^{p}\left(\Omega, \mathbb{R}^{3 \times 3}\right)$, we get

$$
h_{j}^{-2} \int_{\Omega} g_{p}\left(\left|\mathbf{I}+h_{j} \nabla \mathbf{v}_{j}-\mathbf{R}_{j}\right|\right) \mathrm{d} x \leq C
$$

Due to the representation (3.2) of rotations, for every $j \in \mathbb{N}$ there exist $\vartheta_{j} \in(-\pi, \pi]$ and $\mathbf{W}_{j} \in \mathbb{R}_{\text {skew }}^{3 \times 3}$, with $\left|\mathbf{W}_{j}\right|^{2}=2$, such that

$$
\mathbf{R}_{j}=\exp \left(\vartheta_{j} \mathbf{W}_{j}\right)=\mathbf{I}+\sin \vartheta_{j} \mathbf{W}_{j}+\left(1-\cos \vartheta_{j}\right) \mathbf{W}_{j}^{2} .
$$

Hence, by (5.1) and (2.2), we have

$$
\int_{\Omega} g_{p}\left(\left|h_{j} \nabla \mathbf{v}_{j}-\sin \vartheta_{j} \mathbf{W}_{j}-\left(1-\cos \vartheta_{j}\right) \mathbf{W}_{j}^{2}\right|\right) \mathrm{d} x \leq C h_{j}^{2} .
$$

By setting

$$
A_{j}:=\left\{x \in \Omega:\left|h_{j} \nabla \mathbf{v}_{j}-\sin \vartheta_{j} \mathbf{W}_{j}-\left(1-\cos \vartheta_{j}\right) \mathbf{W}_{j}^{2}\right| \leq 1\right\},
$$

from (5.2) we get via Hölder inequality

$$
\begin{aligned}
& \int_{A_{j}}\left|h_{j} \nabla \mathbf{v}_{j}-\sin \vartheta_{j} \mathbf{W}_{j}-\left(1-\cos \vartheta_{j}\right) \mathbf{W}_{j}^{2}\right|^{p} \mathrm{~d} x \\
& \quad \leq\left\{\int_{A_{j}}\left|h_{j} \nabla \mathbf{v}_{j}-\sin \vartheta_{j} \mathbf{W}_{j}-\left(1-\cos \vartheta_{j}\right) \mathbf{W}_{j}^{2}\right|^{2} \mathrm{~d} x\right\}^{p / 2}\left|A_{j}\right|^{1-\frac{p}{2}} \leq C h_{j}^{p},
\end{aligned}
$$

and again by (2.2) we also obtain

$$
\int_{\Omega \backslash A_{j}}\left|h_{j} \nabla \mathbf{v}_{j}-\sin \vartheta_{j} \mathbf{W}_{j}-\left(1-\cos \vartheta_{j}\right) \mathbf{W}_{j}^{2}\right|^{p} \mathrm{~d} x \leq C h_{j}^{2} \leq C h_{j}^{p} .
$$

Since

$$
\operatorname{sym}\left(h_{j} \nabla \mathbf{v}_{j}-\sin \vartheta_{j} \mathbf{W}_{j}-\left(1-\cos \vartheta_{j}\right) \mathbf{W}_{j}^{2}\right)=h_{j} \mathbb{E}\left(\mathbf{v}_{j}\right)-\left(1-\cos \vartheta_{j}\right) \mathbf{W}_{j}^{2},
$$

we get

$$
\int_{\Omega}\left|\mathbb{E}\left(\mathbf{v}_{j}\right)-\left(1-\cos \vartheta_{j}\right) h_{j}^{-1} \mathbf{W}_{j}^{2}\right|^{p} \mathrm{~d} x \leq C .
$$

By recalling that $\mathbb{E}\left(\mathbf{v}_{j}\right) \rightarrow \mathbb{E}(\mathbf{v})$ in $L^{p}\left(\Omega, \mathbb{R}^{3 \times 3}\right)$, we get

$$
\left|1-\cos \vartheta_{j}\right| \leq C h_{j}
$$

hence

$$
\left|\sin \vartheta_{j}\right| \leq \sqrt{2 C h_{j}}
$$

By (5.3) and (5.4), we have

$$
\int_{\Omega}\left|\sqrt{h_{j}} \nabla \mathbf{v}_{j}-h_{j}^{-1 / 2} \sin \vartheta_{j} \mathbf{W}_{j}-h_{j}^{-1 / 2}\left(1-\cos \vartheta_{j}\right) \mathbf{W}_{j}^{2}\right|^{p} \mathrm{~d} x \leq C h_{j}^{p / 2},
$$

therefore, by (5.5) and (5.6) there exists a constant matrix $\mathbf{W} \in \mathbb{R}_{\text {skew }}^{3 \times 3}$ such that, up to subsequences, $\sqrt{h_{j}} \nabla \mathbf{v}_{j} \rightarrow \mathbf{W}$ in $L^{p}\left(\Omega, \mathbb{R}^{3 \times 3}\right)$ as $j \rightarrow \infty$. 


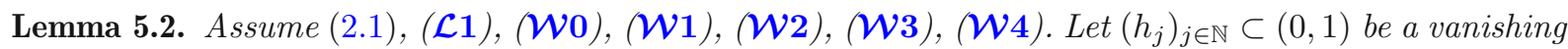
sequence and let $\left(\mathbf{v}_{j}\right)_{j \in \mathbb{N}} \subset W^{1, p}\left(\Omega, \mathbb{R}^{3}\right)$ be a sequence such that $\sup _{j \in \mathbb{N}} \mathcal{F}_{h_{j}}^{I}\left(\mathbf{v}_{j}\right)<+\infty$ and $\mathbb{E}\left(\mathbf{v}_{j}\right) \rightarrow \mathbb{E}(\mathbf{v})$ weakly in $L^{p}\left(\Omega, \mathbb{R}^{3 \times 3}\right)$ as $j \rightarrow \infty$ for some $\mathbf{v} \in W^{1, p}\left(\Omega, \mathbb{R}^{3}\right)$. Then, $\mathbf{v} \in H^{1}\left(\Omega, \mathbb{R}^{3}\right)$ and up to subsequences $\mathbf{1}_{B_{j}} \mathbb{E}\left(\mathbf{v}_{j}\right) \rightarrow \mathbb{E}(\mathbf{v})$ weakly in $L^{2}\left(\Omega, \mathbb{R}^{3 \times 3}\right)$ as $j \rightarrow \infty$, where

$$
B_{j}:=\left\{x \in \Omega:\left|\sqrt{h}_{j} \nabla \mathbf{v}_{j}\right| \leq 2|\boldsymbol{W}|+1\right\}
$$

and where $\boldsymbol{W} \in \mathbb{R}_{\text {skew }}^{3 \times 3}$ is given by Lemma 5.1 .

Proof. Again, through the proof we denote by $C$ the various positive constants, possibly depending only on $\Omega, p, \mathbf{f}, \mathbf{g}$. Let $\theta_{j}$ and $\mathbf{W}_{j}$ be as in the proof of Lemma 5.1 , so that (5.5) holds. We claim that $\mathbf{1}_{B_{j}} \mathbb{E}\left(\mathbf{v}_{j}\right)$ is bounded in $L^{2}\left(\Omega, \mathbb{R}^{3 \times 3}\right)$. To this aim, it is useful to notice that by $(2.2)$ for every $\delta>0$ there exists $c=c(p, \delta)>0$ such that $g_{p}(t) \geq c t^{2}$ for every $t \in[0, \delta]$. Therefore, by taking into account that for $j$ large enough

$$
\mathbf{1}_{B_{j}}\left|h_{j} \mathbb{E}\left(\mathbf{v}_{j}\right)-\left(1-\cos \theta_{j}\right) \mathbf{W}_{j}^{2}\right| \leq 2|\mathbf{W}|+1 .
$$

We may fix $\delta=2|\mathbf{W}|+1$ and obtain for any $j$ large enough ( since $|\operatorname{sym} \mathbf{F}| \leq|\mathbf{F}|$ and $g_{p}$ is increasing)

$$
\begin{aligned}
& \int_{B_{j}}\left|\mathbb{E}\left(\mathbf{v}_{j}\right)\right|^{2} \mathrm{~d} x \leq 2 h_{j}^{-2} \int_{B_{j}}\left|h_{j} \mathbb{E}\left(\mathbf{v}_{j}\right)-\left(1-\cos \theta_{j}\right) \mathbf{W}_{j}^{2}\right|^{2} \mathrm{~d} x+2\left|B_{j}\right| h_{j}^{-2}\left|\left(1-\cos \theta_{j}\right) \mathbf{W}_{j}^{2}\right|^{2} \\
& \quad \leq C h_{j}^{-2} \int_{B_{j}} g_{p}\left(\left|h_{j} \mathbb{E}\left(\mathbf{v}_{j}\right)-\left(1-\cos \theta_{j}\right) \mathbf{W}_{j}^{2}\right|\right) \mathrm{d} x+2\left|B_{j}\right| h_{j}^{-2}\left|\left(1-\cos \theta_{j}\right) \mathbf{W}_{j}^{2}\right|^{2} \\
& \quad \leq C h_{j}^{-2} \int_{B_{j}} g_{p}\left(\left|h_{j} \nabla \mathbf{v}_{j}-\left(1-\cos \theta_{j}\right) \mathbf{W}_{j}^{2}-\sin \theta_{j} \mathbf{W}_{j}\right|\right) \mathrm{d} x+2 h_{j}{ }^{-2}\left|B_{j}\right|\left|\left(1-\cos \theta_{j}\right) \mathbf{W}_{j}^{2}\right|^{2}
\end{aligned}
$$

and by arguing as in the proof of Lemma 5.1, see (5.2) and (5.5), we get

$$
\int_{B_{j}}\left|\mathbb{E}\left(\mathbf{v}_{j}\right)\right|^{2} \mathrm{~d} x \leq C
$$

as claimed. On the other hand, for every $q \in(1, p)$ we have

$$
\int_{B_{j}^{c}}\left|\mathbb{E}\left(\mathbf{v}_{j}\right)\right|^{q} \mathrm{~d} x \leq\left(\int_{B_{j}^{c}}\left|\mathbb{E}\left(\mathbf{v}_{j}\right)\right|^{p} \mathrm{~d} x\right)^{q / p}\left|B_{j}^{c}\right|^{(p-q) / p} \rightarrow 0
$$

since $\left|B_{j}^{c}\right| \rightarrow 0$ by Chebyshev inequality and by Lemma 5.1. By taking into account that

$$
\mathbb{E}\left(\mathbf{v}_{j}\right)=\mathbf{1}_{B_{j}^{c}} \mathbb{E}\left(\mathbf{v}_{j}\right)+\mathbf{1}_{B_{j}} \mathbb{E}\left(\mathbf{v}_{j}\right)
$$

and by assuming wlog that $\mathbf{1}_{B_{j}} \mathbb{E}\left(\mathbf{v}_{j}\right) \rightarrow \mathbf{u}$ weakly in $L^{2}\left(\Omega, \mathbb{R}^{3 \times 3}\right)$ we get $\mathbb{E}\left(\mathbf{v}_{j}\right) \rightarrow \mathbf{u}$ weakly in $L^{q}\left(\Omega, \mathbb{R}^{3 \times 3}\right)$ and recalling that $\mathbb{E}\left(\mathbf{v}_{j}\right) \rightarrow \mathbb{E}(\mathbf{v})$ weakly in $L^{p}\left(\Omega, \mathbb{R}^{3 \times 3}\right)$ we get $\mathbf{u}=\mathbb{E}(\mathbf{v}) \in L^{2}\left(\Omega, \mathbb{R}^{3 \times 3}\right)$ thus proving that $\mathbf{v} \in H^{1}\left(\Omega, \mathbb{R}^{3}\right)$ by Korn inequality.

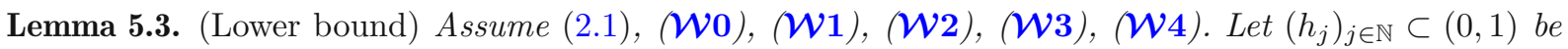
a vanishing sequence and let $\left(\mathbf{v}_{j}\right)_{j \in \mathbb{N}} \subset W^{1, p}\left(\Omega, \mathbb{R}^{3}\right)$ be a sequence such that $\mathbb{E}\left(\mathbf{v}_{j}\right) \rightarrow \mathbb{E}(\mathbf{v})$ weakly in $L^{p}\left(\Omega, \mathbb{R}^{3 \times 3}\right)$ as $j \rightarrow \infty$. Then,

$$
\liminf _{j \rightarrow+\infty} \mathcal{F}_{h_{j}}^{I}\left(\mathbf{v}_{j}\right) \geq \mathcal{F}^{I}(\mathbf{v}) .
$$


Proof. We may assume wlog that $\mathcal{F}_{h_{j}}^{I}\left(\mathbf{v}_{j}\right) \leq C$ for any $j \in \mathbb{N}$ so that by setting

$$
\mathbf{D}_{j}:=\mathbb{E}\left(\mathbf{v}_{j}\right)+\frac{1}{2} h_{j} \nabla \mathbf{v}_{j}^{T} \nabla \mathbf{v}_{j}
$$

we get

$$
\begin{aligned}
1 & =\operatorname{det}\left(\mathbf{I}+h_{j} \nabla \mathbf{v}_{j}\right)=\operatorname{det}\left(\mathbf{I}+h_{j} \nabla \mathbf{v}_{j}^{T}\right)\left(\mathbf{I}+h_{j} \nabla \mathbf{v}_{j}\right)=\operatorname{det}\left(\mathbf{I}+2 h_{j} \mathbb{E}\left(\mathbf{v}_{j}\right)+h_{j}^{2} \nabla \mathbf{v}_{j}^{T} \nabla \mathbf{v}_{j}\right) \\
& =1+2 h_{j} \operatorname{Tr} \mathbf{D}_{j}-2 h_{j}^{2}\left(\operatorname{Tr}\left(\mathbf{D}_{j}^{2}\right)-\left(\operatorname{Tr} \mathbf{D}_{j}\right)^{2}\right)+8 h_{j}^{3} \operatorname{det} \mathbf{D}_{j}
\end{aligned}
$$

a.e. in $\Omega$, that is,

$$
2 \operatorname{div} \mathbf{v}_{j}+h_{j}\left|\nabla \mathbf{v}_{j}\right|^{2}=2 \operatorname{Tr} \mathbf{D}_{j}=2 h_{j}\left(\operatorname{Tr}\left(\mathbf{D}_{j}^{2}\right)-\left(\operatorname{Tr} \mathbf{D}_{j}\right)^{2}\right)-8 h_{j}^{2} \operatorname{det} \mathbf{D}_{j} .
$$

By taking into account Lemma (5.1) we get $\sqrt{h}_{j} \nabla \mathbf{v}_{j} \rightarrow \mathbf{W}$ in $L^{p}$ hence, up to subsequences, $h_{j} \nabla \mathbf{v}_{j}^{T} \nabla \mathbf{v}_{j} \rightarrow$ $-\mathbf{W}^{2}$ a.e. in $\Omega$ and $2 h_{j}\left(\operatorname{Tr}\left(\mathbf{D}_{j}^{2}\right)-\left(\operatorname{Tr} \mathbf{D}_{j}\right)^{2}\right)-8 h_{j}^{2} \operatorname{det} \mathbf{D}_{j} \rightarrow 0$ a.e. in $\Omega$. Therefore, $2 \operatorname{div} \mathbf{v}_{j} \rightarrow \operatorname{Tr} \mathbf{W}^{2}$ a.e. in $\Omega$ and since the weak convergence of $\mathbb{E}\left(\mathbf{v}_{j}\right)$ implies $\operatorname{div} \mathbf{v}_{j} \rightarrow \operatorname{div} \mathbf{v}$ weakly in $L^{p}(\Omega)$ we get $2 \operatorname{div} \mathbf{v}=\operatorname{Tr} \mathbf{W}^{2}$ a.e. in $\Omega$. On the other hand by Lemma 5.2 , with $B_{j}$ defined by (5.7), we have $\mathbf{1}_{B_{j}} \mathbb{E}\left(\mathbf{v}_{j}\right) \rightarrow \mathbb{E}(\mathbf{v})$ weakly in $L^{2}\left(\Omega, \mathbb{R}^{3 \times 3}\right)$ and $\mathbf{v} \in H^{1}\left(\Omega, \mathbb{R}^{3}\right)$. Hence, by $(3.7),(3.4)$ and $(\mathcal{L} 1)$ we get for large enough $j$

$$
\begin{aligned}
\mathcal{F}_{h_{j}}^{I}\left(\mathbf{v}_{j}\right) & \geq \frac{1}{h_{j}^{2}} \int_{B_{j}} \mathcal{W}\left(x, \mathbf{I}+h_{j} \nabla \mathbf{v}_{j}\right) \mathrm{d} x-\mathcal{L}\left(\mathbf{v}_{j}-\mathbb{P} \mathbf{v}_{j}\right)=\frac{1}{h_{j}^{2}} \int_{B_{j}} \mathcal{V}\left(x, h_{j} \mathbf{D}_{j}\right) \mathrm{d} x-\mathcal{L}\left(\mathbf{v}_{j}-\mathbb{P} \mathbf{v}_{j}\right) \\
& \geq \int_{B_{j}} \frac{1}{2} \mathbf{D}_{j}^{T} D^{2} \mathcal{V}(x, \mathbf{0}) \mathbf{D}_{j} \mathrm{~d} x-\int_{B_{j}} \eta\left(h_{j} \mathbf{D}_{j}\right)\left|\mathbf{D}_{j}\right|^{2} \mathrm{~d} x-\mathcal{L}\left(\mathbf{v}_{j}-\mathbb{P}_{j}\right),
\end{aligned}
$$

since on $B_{j}$ we have $h_{j}\left|\mathbf{D}_{j}\right| \leq \sqrt{h_{j}}\left(\sqrt{h_{j}}\left|\nabla \mathbf{v}_{j}\right|+\frac{1}{2} h_{j}^{3 / 2}\left|\nabla \mathbf{v}_{j}^{T}\right|\left|\nabla \mathbf{v}_{j}\right|\right) \leq \sqrt{h_{j}}(2|\mathbf{W}|+2)$ for large enough $j$ ( so that indeed (3.7) can be applied). We deduce

$$
\begin{aligned}
\mathcal{F}_{h_{j}}^{I}\left(\mathbf{v}_{j}\right) \geq \frac{1}{2} & \int_{\Omega}\left(\mathbf{1}_{B_{j}} \mathbf{D}_{j}\right)^{T} D^{2} \mathcal{W}(x, \mathbf{I})\left(\mathbf{1}_{B_{j}} \mathbf{D}_{j}\right) \mathrm{d} x \\
& -\eta\left(\sqrt{h}_{j}(2|\mathbf{W}|+1) \int_{\Omega}\left|\mathbf{1}_{B_{j}} \mathbf{D}_{j}\right|^{2} \mathrm{~d} x-\mathcal{L}\left(\mathbf{v}_{j}-\mathbb{P} \mathbf{v}_{j}\right)\right.
\end{aligned}
$$

for large enough $j$, as $\eta$ is increasing. Since $h_{j} \nabla \mathbf{v}_{j}^{T} \nabla \mathbf{v}_{j} \rightarrow-\mathbf{W}^{2}$ a.e. in $\Omega$ and $\left|B_{j}^{c}\right| \rightarrow 0$ as $j \rightarrow+\infty$, and since $\left|\mathbf{1}_{B_{j}} h_{j} \nabla \mathbf{v}_{j}^{T} \nabla \mathbf{v}_{j}\right| \leq(2|\mathbf{W}|+1)^{2}$, we get $\mathbf{1}_{B_{j}} h_{j} \nabla \mathbf{v}_{j}^{T} \nabla \mathbf{v}_{j} \rightarrow-\mathbf{W}^{2}$ weakly in $L^{2}\left(\Omega, \mathbf{R}^{3 \times 3}\right)$. By taking into account that $\mathbf{1}_{B_{j}} \mathbb{E}\left(\mathbf{v}_{j}\right) \rightarrow \mathbb{E}(\mathbf{v})$ weakly in $L^{2}\left(\Omega, \mathbf{R}^{3 \times 3}\right)$, we then obtain $\mathbf{1}_{B_{j}} \mathbf{D}_{j} \rightarrow \mathbb{E}(\mathbf{v})-\frac{1}{2} \mathbf{W}^{2}$ weakly in $L^{2}\left(\Omega, \mathbb{R}^{3 \times 3}\right)$. Hence, by $(\mathcal{W} \mathbf{3}),(5.9)$ and by the weak $L^{2}\left(\Omega, \mathbb{R}^{3 \times 3}\right)$ lower semicontinuity of the map $\mathbf{F} \mapsto \int_{\Omega} \mathbf{F}^{T} D^{2} \mathcal{W}(x, \mathbf{I}) \mathbf{F} \mathrm{d} x$, we deduce

$$
\liminf _{j \rightarrow+\infty} \mathcal{F}_{h_{j}}^{I}\left(\mathbf{v}_{j}\right) \geq \frac{1}{2} \int_{\Omega}\left(\mathbb{E}(\mathbf{v})-\frac{1}{2} \mathbf{W}^{2}\right) D^{2} \mathcal{W}(x, \mathbf{I})\left(\mathbb{E}(\mathbf{v})-\frac{1}{2} \mathbf{W}^{2}\right) \mathrm{d} x-\mathcal{L}(\mathbf{v}) .
$$

In order to obtain (5.10), we have also used the fact that $\mathcal{L}\left(\mathbf{v}_{j}-\mathbb{P} \mathbf{v}_{j}\right) \rightarrow \mathcal{L}(\mathbf{v})$ as $j \rightarrow+\infty$. Indeed, by (3.9) and (3.10) we obtain boundedness of $\mathbf{v}_{j}-\mathbb{P} \mathbf{v}_{j}$ in $W^{1, p}\left(\Omega, \mathbb{R}^{3}\right)$ and in $L^{\frac{3 p}{3-p}}\left(\Omega, \mathbb{R}^{3}\right)$. Therefore, up to subsequences we get $\mathbf{v}_{j}-\mathbb{P} \mathbf{v}_{j} \rightarrow \mathbf{w}$ weakly in $L^{\frac{3 p}{3-p}}\left(\Omega, \mathbb{R}^{3}\right)$ for some $\mathbf{w} \in W^{1, p}\left(\Omega, \mathbb{R}^{3}\right)$, and by trace embedding $\mathbf{v}_{j}-\mathbb{P} \mathbf{v}_{j} \rightarrow \mathbf{w}$ weakly in $L^{\frac{2 p}{3-p}}\left(\partial \Omega, \mathbb{R}^{3}\right)$ as well. Moreover, since $\mathbb{E}\left(\mathbf{v}_{j}-\mathbb{P} \mathbf{v}_{j}\right) \rightarrow \mathbb{E}(\mathbf{v})$ weakly in $L^{p}\left(\Omega, \mathbb{R}^{3 \times 3}\right)$, we deduce $\mathbb{E}(\mathbf{w})=\mathbb{E}(\mathbf{v})$, thus $(\mathcal{L} 1)$ implies $\mathcal{L}(\mathbf{w})=\mathcal{L}(\mathbf{v})$. Therefore, we have $\mathcal{L}\left(\mathbf{v}_{j}-\mathbb{P} \mathbf{v}_{j}\right) \rightarrow \mathcal{L}(\mathbf{w})=\mathcal{L}(\mathbf{v})$ as $j \rightarrow+\infty$.

Eventually, since $2 \operatorname{div} \mathbf{v}=\operatorname{Tr} \mathbf{W}^{2}$ a.e. in $\Omega$, the result follows from (5.10). 


\section{Upper bound}

The following result is an extension of [25, Lemma 4.1].

Lemma 6.1. Let $\Omega$ satisfy assumption (2.1). Let $\mathbf{v} \in C^{1}\left(\Omega^{\prime}, \mathbb{R}^{3}\right) \cap W^{2, \infty}\left(\Omega^{\prime}, \mathbb{R}^{3}\right)$ be such that $\operatorname{div} \mathbf{v}=0$ in $\Omega^{\prime}$, where $\Omega^{\prime} \subset \mathbb{R}^{3}$ is an open set such that $\bar{\Omega} \subset \Omega^{\prime}$. Let $\left(h_{j}\right)_{j \in \mathbb{N}} \subset(0,1)$ be a vanishing sequence. There exists a sequence of vector fields $\left(\mathbf{v}_{j}\right)_{j \in \mathbb{N}} \subset C^{1}\left(\Omega, \mathbb{R}^{3}\right) \cap W^{2, \infty}\left(\Omega, \mathbb{R}^{3}\right)$ and $j_{*} \in \mathbb{N}$ such that for any $j>j_{*}$

$$
\begin{aligned}
& \operatorname{det}\left(\boldsymbol{I}+h_{j} \nabla \mathbf{v}_{j}\right)=1, \\
& \sup _{x \in \Omega}\left|\mathbf{v}_{j}(x)-\mathbf{v}(x)\right| \leq\|\mathbf{v}\|_{L^{\infty}(\Omega)} \mathfrak{q}\left(h_{j}\|\mathbf{v}\|_{W^{1, \infty}(\Omega)}\right), \\
& \sup _{x \in \Omega}\left|h_{j} \nabla \mathbf{v}_{j}(x)\right| \leq \mathfrak{q}\left(h_{j}\|\mathbf{v}\|_{W^{1, \infty}(\Omega)}\right), \\
& \sup _{x \in \Omega}\left|\nabla \mathbf{v}_{j}(x)-\nabla \mathbf{v}(x)\right| \leq\left(1+e^{h_{j}\|\mathbf{v}\|_{W^{1, \infty}\left(\Omega^{\prime}\right)}}\right)\|\mathbf{v}\|_{W^{2, \infty}\left(\Omega^{\prime}\right)} \mathfrak{q}\left(h_{j}\|\mathbf{v}\|_{W^{1, \infty}\left(\Omega^{\prime}\right)}\right),
\end{aligned}
$$

where $\mathfrak{q}(z):=z e^{z}$. In particular, $\mathbf{v}_{j} \rightarrow \mathbf{v}$ in $W^{1, \infty}\left(\Omega, \mathbb{R}^{3}\right)$ as $j \rightarrow+\infty$.

Proof. We choose $T \in(0,1)$ small enough, such that $\mathbf{y}(t, x) \in \Omega^{\prime}$ for any $x \in \Omega$ and any $t \in[0, T]$, where $\mathbf{y}(\cdot, x)$ is the unique solution to

$$
\left\{\begin{array}{l}
\frac{\partial \mathbf{y}}{\partial t}(t, x)=\mathbf{v}(\mathbf{y}(t, x)), \quad t \in(0, T] \\
\mathbf{y}(0, x)=x
\end{array}\right.
$$

so that $\mathbf{y}$ is the flow associated to the vector field $\mathbf{v}$. We have $\mathbf{y} \in C^{1}\left([0, T] ; W^{2, \infty}(\Omega)\right)$, see $[15$, Corollary 5.2.8, Remark 5.2.9]. From (6.5), we have

$$
\frac{1}{t}(\mathbf{y}(t, x)-x)-\mathbf{v}(x)=\frac{1}{t} \int_{0}^{t}(\mathbf{v}(\mathbf{y}(s, x))-\mathbf{v}(x)) \mathrm{d} s
$$

for any $x \in \Omega$. We get therefore the basic estimate

$$
\frac{1}{t}|\mathbf{y}(t, x)-x| \leq|\mathbf{v}(x)|+\|\mathbf{v}\|_{W^{1, \infty}\left(\Omega^{\prime}\right)} \int_{0}^{t} \frac{1}{s}|\mathbf{y}(s, x)-x| \mathrm{d} s
$$

for any $x \in \Omega$, and Gronwall lemma entails

$$
\frac{1}{t}|\mathbf{y}(t, x)-x| \leq|\mathbf{v}(x)| \exp \left\{\|\mathbf{v}\|_{W^{1, \infty}\left(\Omega^{\prime}\right)} t\right\},
$$

so that we have

$$
\sup _{x \in \Omega}|\mathbf{y}(t, x)-x| \leq \mathfrak{q}\left(t\|\mathbf{v}\|_{W^{1, \infty}\left(\Omega^{\prime}\right)}\right) .
$$

We have $\nabla \mathbf{y} \in C^{1}\left([0, T] ; W^{1, \infty}(\Omega)\right)$, where $\nabla$ denotes the gradient in $x$, and as shown in the proof of $[25$, Lemma 4.1], there hold

$$
\nabla \mathbf{y}(t, x)=\exp \left(\int_{0}^{t} \nabla \mathbf{v}(\mathbf{y}(s, x)) \mathrm{d} s\right)
$$

and then

$$
\operatorname{det} \nabla \mathbf{y}(t, x)=\exp \left(\int_{0}^{t} \operatorname{Tr} \nabla \mathbf{v}(\mathbf{y}(s, x)) \mathrm{d} s\right)=\exp \left(\int_{0}^{t} \operatorname{div} \mathbf{v}(\mathbf{y}(s, x)) \mathrm{d} s\right)=1
$$


for every $t \in(0, T]$ and for every $x \in \Omega$.

We define

$$
\mathbf{v}_{t}(x):=t^{-1}(\mathbf{y}(t, x)-x), \quad x \in \Omega, t \in(0, T] .
$$

From the definition of $\mathbf{v}_{t}$, from (6.6) and (6.7) we get

$$
\begin{aligned}
\left|\mathbf{v}_{t}(x)-\mathbf{v}(x)\right| & =\left|\frac{1}{t}(\mathbf{y}(t, x)-x)-\mathbf{v}(x)\right| \leq\|\mathbf{v}\|_{W^{1, \infty}(\Omega)} \int_{0}^{t} \frac{1}{s}|\mathbf{y}(s, x)-x| \mathrm{d} s \\
& \leq\|\mathbf{v}\|_{L^{\infty}(\Omega)} \mathfrak{q}\left(t\|\mathbf{v}\|_{W^{1, \infty}(\Omega)}\right)
\end{aligned}
$$

for any $x \in \Omega$ and any $t \in(0, T]$. From the latter, we get in particular the convergence of $\mathbf{v}_{t}$ to $\mathbf{v}$ in $L^{1} \cap L^{\infty}(\Omega)$ as $t \rightarrow 0$.

Since the map $\Omega \ni x \mapsto \mathbf{v}(\mathbf{y}(t, x))$ is Lipschitz continuous, uniformly with respect to $t \in(0, T)$, we may take the gradient under integral sign in (6.6) and obtain

$$
\begin{aligned}
\frac{1}{t}(\nabla \mathbf{y}(t, x)-\mathbf{I})-\nabla \mathbf{v}(x)= & \frac{1}{t} \int_{0}^{t}(\nabla[\mathbf{v}(\mathbf{y}(s, x))]-\nabla \mathbf{v}(x)) \mathrm{d} s \\
= & \frac{1}{t} \int_{0}^{t}(\nabla \mathbf{v}(\mathbf{y}(s, x)) \nabla \mathbf{y}(s, x)-\nabla \mathbf{v}(x) \nabla \mathbf{y}(s, x)) \mathrm{d} s \\
& +\frac{1}{t} \int_{0}^{t}(\nabla \mathbf{v}(x) \nabla \mathbf{y}(s, x)-\nabla \mathbf{v}(x)) \mathrm{d} s
\end{aligned}
$$

for every $x \in \Omega$ and every $t \in(0, T]$. From the first equality of (6.12) and from (6.9), we get

$$
\begin{aligned}
\frac{1}{t}|\nabla \mathbf{y}(t, x)-\mathbf{I}| & \leq \frac{1}{t} \int_{0}^{t}|\nabla \mathbf{v}(\mathbf{y}(s, x))||\nabla \mathbf{y}(s, x)| \mathrm{d} s \\
& \leq\|\mathbf{v}\|_{W^{1, \infty}\left(\Omega^{\prime}\right)} \frac{1}{t} \int_{0}^{t}|\nabla \mathbf{y}(s, x)| \mathrm{d} s \leq\|\mathbf{v}\|_{W^{1, \infty}\left(\Omega^{\prime}\right)} \frac{1}{t} \int_{0}^{t} \exp \left\{s\|\mathbf{v}\|_{W^{1, \infty}\left(\Omega^{\prime}\right)}\right\} \mathrm{d} s \\
& \leq\|\mathbf{v}\|_{W^{1, \infty}\left(\Omega^{\prime}\right)} \exp \left\{t\|\mathbf{v}\|_{W^{1, \infty}\left(\Omega^{\prime}\right)}\right\},
\end{aligned}
$$

therefore

$$
\sup _{x \in \Omega}|\nabla \mathbf{y}(t, x)-\mathbf{I}| \leq \mathfrak{q}\left(t\|\mathbf{v}\|_{W^{1, \infty}\left(\Omega^{\prime}\right)}\right)
$$


for any $t \in(0, T]$. Moreover, by (6.12), (6.8), (6.13) and (6.9) we have

$$
\begin{aligned}
& \left|\nabla \mathbf{v}_{t}(x)-\nabla \mathbf{v}(x)\right|=\left|\frac{1}{t}(\nabla \mathbf{y}(t, x)-\mathbf{I})-\nabla \mathbf{v}(x)\right| \\
& \quad \leq \frac{1}{t} \int_{0}^{t}|\nabla \mathbf{y}(s, x)||\nabla \mathbf{v}(\mathbf{y}(s, x))-\nabla \mathbf{v}(x)| \mathrm{d} s+\|\mathbf{v}\|_{W^{1, \infty}\left(\Omega^{\prime}\right)} \frac{1}{t} \int_{0}^{t}|\nabla \mathbf{y}(s, x)-\mathbf{I}| \mathrm{d} s \\
& \quad \leq\|\mathbf{v}\|_{W^{2, \infty}\left(\Omega^{\prime}\right)} \frac{1}{t} \int_{0}^{t}|\nabla \mathbf{y}(s, x)||\mathbf{y}(s, x)-x| \mathrm{d} s+\|\mathbf{v}\|_{W^{1, \infty}\left(\Omega^{\prime}\right)} \mathfrak{q}\left(t\|\mathbf{v}\|_{W^{1, \infty}\left(\Omega^{\prime}\right)}\right) \\
& \quad \leq\|\mathbf{v}\|_{W^{2, \infty}\left(\Omega^{\prime}\right)} \frac{1}{t} \int_{0}^{t} \exp \left\{s\|\mathbf{v}\|_{W^{1, \infty}\left(\Omega^{\prime}\right)}\right\} \mathfrak{q}\left(s\|\mathbf{v}\|_{W^{1, \infty}\left(\Omega^{\prime}\right)}\right) \mathrm{d} s+\|\mathbf{v}\|_{W^{1, \infty}\left(\Omega^{\prime}\right)} \mathfrak{q}\left(t\left\|_{\mathbf{v}}\right\|_{W^{1, \infty}\left(\Omega^{\prime}\right)}\right) \\
& \quad \leq\left(e^{\left.t\|\mathbf{v}\|_{W^{1, \infty}\left(\Omega^{\prime}\right)}\right)}\|\mathbf{v}\|_{W^{2, \infty}\left(\Omega^{\prime}\right)} \mathfrak{q}\left(t\|\mathbf{v}\|_{W^{1, \infty}\left(\Omega^{\prime}\right)}\right)\right.
\end{aligned}
$$

for any $x \in \Omega$ and any $t \in(0, T]$.

Eventually, let us consider a vanishing sequence $\left(h_{j}\right)_{j \in \mathbb{N}} \subset(0,1)$. By defining $j_{*}$ as the smallest positive integer such that $h_{j}<T$ for any $j>j_{*}$ and by defining $\mathbf{v}_{j}:=\mathbf{v}_{h_{j}}$, the result follows from (6.10), (6.11), (6.13) and from the latter estimate.

We next provide the approximation construction for the recovery sequence.

Lemma 6.2. Let $\Omega$ satisfy assumption $(2.1)$. Let $\left(h_{j}\right)_{j \in \mathbb{N}} \subset(0,1)$ be a vanishing sequence. Let $\mathbf{v} \in H_{\text {div }}^{1}(\Omega)$. There exists a sequence $\left(\mathbf{v}_{j}\right)_{j \in \mathbb{N}} \subset W^{2, \infty}\left(\Omega, \mathbb{R}^{3}\right)$ such that

(i) $\operatorname{det}\left(\boldsymbol{I}+h_{j} \nabla \mathbf{v}_{j}\right)=1$ for any $j \in \mathbb{N}$,

(ii) $h_{j}\left\|\nabla \mathbf{v}_{j}\right\|_{L^{\infty}(\Omega)} \rightarrow 0$ as $j \rightarrow+\infty$,

(iii) $\mathbf{v}_{j} \rightarrow \mathbf{v}$ strongly in $H^{1}\left(\Omega, \mathbb{R}^{3}\right)$ as $j \rightarrow+\infty$.

Proof. Assumption (2.1) implies that there are $m$ bounded open connected Lipschitz sets with connected boundary, denoted by $\Omega_{*}, \Omega_{1}, \ldots, \Omega_{m-1}$, such that $\Omega=\Omega_{*} \backslash\left(\bar{\Omega}_{1} \cup \cdots \cup \cdots \bar{\Omega}_{m-1}\right)$. We have $\Omega_{*} \equiv \Omega$ if $m=1$. By [20, Corollary 3.2], we may extend $\mathbf{v}$ to a $H^{1}\left(\mathbb{R}^{3} \backslash \overline{P_{\delta}}, \mathbb{R}^{3}\right)$ vector field, still denoted by $\mathbf{v}$, such that $\operatorname{div} \mathbf{v}=0$ on $\mathbb{R}^{3} \backslash P_{\delta}$, where $P:=\left\{p_{1}, \ldots, p_{m-1}\right\}, p_{i} \in \Omega_{i}$ for any $i=1, \ldots, m-1$, and $P_{\delta}:=B_{\delta}\left(p_{1}\right) \cup \cdots \cup B_{\delta}\left(p_{m-1}\right)$. We have $P=P_{\delta}=\emptyset$ if $m=1$, otherwise $\delta>0$ is fixed and so small that $\overline{B_{\delta}\left(p_{i}\right)} \subset \Omega_{i}$ for any $i=1, \ldots, m-1$. We note that this extension can be constructed in the form $\mathbf{v}=\operatorname{div} \mathbf{V}+\lambda_{1} \mathbf{g}_{1}+\cdots+\lambda_{m-1} \mathbf{g}_{m-1}$, where $\mathbf{V} \in H^{2}\left(\mathbb{R}^{3}, \mathbb{R}^{3 \times 3}\right), \lambda_{i} \in \mathbb{R}$ and $\mathbf{g}_{i}$ is a harmonic field with singularity at $p_{i}$ for each $i=1, \ldots, m-1$.

Let $\rho$ denote the standard unit symmetric mollifier on $\mathbb{R}^{3}$ and let $\rho_{\varepsilon}(x):=\varepsilon^{-3} \rho(x / \varepsilon)$. We let $\mathbf{v}^{\varepsilon}:=\mathbf{v} * \rho_{\varepsilon}$ and we let $\Omega^{\prime}$ be a bounded open set such that $\bar{\Omega} \subset \Omega^{\prime}$. We may choose $\Omega^{\prime}$ such that $\mathbf{v}^{\varepsilon} \in C^{\infty}\left(\overline{\Omega^{\prime}}\right)$, such that $\bar{\Omega} \subset \Omega^{\prime} \subset \subset \mathbb{R}^{3} \backslash \overline{P_{\delta}}$ and such that $\operatorname{div} \mathbf{v}^{\varepsilon} \equiv 0$ in $\Omega^{\prime}$ for any small enough $\varepsilon$. Therefore, we may fix a constant $c \in(0,1)$, only depending on $\delta$, such that $\varepsilon_{n}:=c / n$ satisfies the above properties for any $n \in \mathbb{N}$.

Given a vanishing sequence $\left(h_{j}\right)_{j \in \mathbb{N}}$ of positive numbers and given $n \in \mathbb{N}$ we may define $\left(\mathbf{v}_{j}^{\varepsilon_{n}}\right)_{j \in \mathbb{N}}$ to be the sequence from Lemma 6.1 , constructed from the $W^{2, \infty}\left(\Omega^{\prime}, \mathbb{R}^{3}\right)$ divergence-free vector field $\mathbf{v}^{\varepsilon_{n}}$ : indeed, by applying Lemma 6.1 to $\mathbf{v}^{\varepsilon_{n}}$, we construct a sequence of $W^{2, \infty}(\Omega)$ vector fields $\left(\mathbf{v}_{j}^{\varepsilon_{n}}\right)_{j \in \mathbb{N}}$ and a strictly increasing diverging function $g: \mathbb{N} \rightarrow \mathbb{N}$ such that (6.1), (6.2), (6.3), (6.4) are satisfied for any 
positive integers $j, n$ with $j>g(n)$, i.e.,

$$
\begin{aligned}
& \operatorname{det}\left(\mathbf{I}+h_{j} \nabla \mathbf{v}_{j}^{\varepsilon_{n}}\right)=1, \\
& \sup _{x \in \Omega}\left|\mathbf{v}_{j}^{\varepsilon_{n}}(x)-\mathbf{v}^{\varepsilon_{n}}(x)\right| \leq\left\|\mathbf{v}^{\varepsilon_{n}}\right\|_{L^{\infty}\left(\Omega^{\prime}\right)} \mathfrak{q}\left(h_{j}\left\|\mathbf{v}^{\varepsilon_{n}}\right\|_{W^{1, \infty}\left(\Omega^{\prime}\right)}\right), \\
& \sup _{x \in \Omega}\left|h_{j} \nabla \mathbf{v}_{j}^{\varepsilon_{n}}(x)\right| \leq \mathfrak{q}\left(h_{j}\left\|\mathbf{v}^{\varepsilon_{n}}\right\|_{W^{1, \infty}\left(\Omega^{\prime}\right)}\right), \\
& \sup _{x \in \Omega}\left|\nabla \mathbf{v}_{j}^{\varepsilon_{n}}(x)-\nabla \mathbf{v}^{\varepsilon_{n}}(x)\right| \leq\left(1+e^{h_{j}\left\|\mathbf{v}^{\varepsilon_{n}}\right\|_{W^{1, \infty}\left(\Omega^{\prime}\right)}}\right)\left\|\mathbf{v}^{\varepsilon_{n}}\right\|_{W^{2, \infty}\left(\Omega^{\prime}\right)} \mathfrak{q}\left(h_{j}\left\|\mathbf{v}^{\varepsilon_{n}}\right\|_{W^{1, \infty}\left(\Omega^{\prime}\right)}\right) .
\end{aligned}
$$

Therefore, by defining $g_{*}(j):=\max \{n \in \mathbb{N}: g(n)<j\}$ for any integer $j$ such that $j>j_{0}:=g(1)$, and by defining the diverging sequence $(n(j))_{j>j_{0}} \subset \mathbb{N}$ as

$$
n(j):=\left\lfloor\min \left\{g_{*}(j), h_{j}^{-1 / 10}\right\}\right\rfloor,
$$

where $\lfloor\cdot\rfloor$ denotes integer part, we obtain that (6.14), (6.15), (6.16), (6.17) are satisfied with $\varepsilon_{n(j)}$ in place of $\varepsilon_{n}$ for any $j>j_{0}$, since $n(j) \leq g_{*}(j)$ (which implies $g(n(j))<j$ ).

We let therefore $\mathbf{v}_{j}:=\mathbf{v}_{j}^{\varepsilon_{n(j)}}$ if $j>j_{0}$ (and, say, $\mathbf{v}_{j} \equiv 0$ if $j=1, \ldots, j_{0}$ ) and conclude by checking that the sequence $\left(\mathbf{v}_{j}\right)_{j \in \mathbb{N}}$ satisfies the desired properties. Property (i) is already given by (6.14). Moreover, by the elementary estimates

$$
\left\|\rho_{\varepsilon}\right\|_{W^{k, \infty}\left(\mathbb{R}^{3}\right)} \leq(k+1) \varepsilon^{-3-k}\|\rho\|_{W^{k, \infty}\left(\mathbb{R}^{3}\right)}, \quad k=0,1,2,
$$

we get

$$
\begin{aligned}
\left\|\mathbf{v}^{\varepsilon_{n(j)}}\right\|_{W^{k, \infty}\left(\Omega^{\prime}\right)} & \leq\|\mathbf{v}\|_{L^{1}\left(\Omega^{\prime}\right)}\left\|\rho_{\varepsilon_{n(j)}}\right\|_{W^{k, \infty}\left(\mathbb{R}^{3}\right)} \leq(k+1) \varepsilon_{n(j)}^{-3-k}\|\mathbf{v}\|_{L^{1}\left(\Omega^{\prime}\right)}\|\rho\|_{W^{k, \infty}\left(\mathbb{R}^{3}\right)}, \\
& =c^{-3-k}(k+1) n(j)^{3+k}\|\mathbf{v}\|_{L^{1}\left(\Omega^{\prime}\right)}\|\rho\|_{W^{k, \infty}\left(\mathbb{R}^{3}\right)}, \quad k=0,1,2,
\end{aligned}
$$

therefore

$$
\begin{aligned}
h_{j}\left\|\mathbf{v}^{\varepsilon_{n(j)}}\right\|_{W^{1,+\infty}\left(\Omega^{\prime}\right)} & \leq 2 h_{j} c^{-4} n(j)^{4}\|\mathbf{v}\|_{L^{1}\left(\Omega^{\prime}\right)}\|\rho\|_{W^{1, \infty}\left(\mathbb{R}^{3}\right)} \\
& \leq 2 c^{-4} h_{j}^{3 / 5}\|\mathbf{v}\|_{L^{1}\left(\Omega^{\prime}\right)}\|\rho\|_{W^{1, \infty}\left(\mathbb{R}^{3}\right)},
\end{aligned}
$$

so that $h_{j}\left\|\mathbf{v}^{\varepsilon_{n(j)}}\right\|_{W^{1,+\infty}\left(\Omega^{\prime}\right)}$ vanishes as $j \rightarrow+\infty$ and then (6.16) implies property (ii). On the other hand, (6.18) similarly implies

$$
h_{j}\left\|\mathbf{v}^{\varepsilon_{n(j)}}\right\|_{W^{1, \infty}\left(\Omega^{\prime}\right)}\left\|\mathbf{v}^{\varepsilon_{n(j)}}\right\|_{L^{\infty}\left(\Omega^{\prime}\right)} \leq 2 c^{-7} h_{j}^{3 / 10}\|\mathbf{v}\|_{L^{1}\left(\Omega^{\prime}\right)}^{2}\|\rho\|_{W^{2, \infty}\left(\mathbb{R}^{3}\right)}^{2}
$$

and

$$
h_{j}\left\|\mathbf{v}^{\varepsilon_{n(j)}}\right\|_{W^{1, \infty}\left(\Omega^{\prime}\right)}\left\|\mathbf{v}^{\varepsilon_{n(j)}}\right\|_{W^{2, \infty}\left(\Omega^{\prime}\right)} \leq 6 c^{-9} h_{j}^{1 / 10}\|\mathbf{v}\|_{L^{1}\left(\Omega^{\prime}\right)}^{2}\|\rho\|_{W^{2, \infty}\left(\mathbb{R}^{3}\right)}^{2} .
$$

Thanks to (6.19), (6.20) and (6.21), from (6.15) and (6.17) we obtain

$$
\lim _{j \rightarrow+\infty}\left\|\mathbf{v}_{j}-\mathbf{v}^{\varepsilon_{n(j)}}\right\|_{W^{1, \infty}(\Omega)}=0 .
$$

This entails, since $\mathbf{v}^{\varepsilon} \rightarrow \mathbf{v}$ in $H^{1}\left(\Omega, \mathbb{R}^{3}\right)$ as $\varepsilon \rightarrow 0$ and since $\varepsilon_{n(j)} \rightarrow 0$ as $j \rightarrow+\infty$,

$$
\lim _{j \rightarrow+\infty}\left\|\mathbf{v}_{j}-\mathbf{v}\right\|_{H^{1}(\Omega)} \leq \lim _{j \rightarrow+\infty}\left\|\mathbf{v}_{j}-\mathbf{v}^{\varepsilon_{n(j)}}\right\|_{H^{1}(\Omega)}+\lim _{j \rightarrow+\infty}\left\|\mathbf{v}-\mathbf{v}^{\varepsilon_{n(j)}}\right\|_{H^{1}(\Omega)}=0
$$

thus proving (iii).

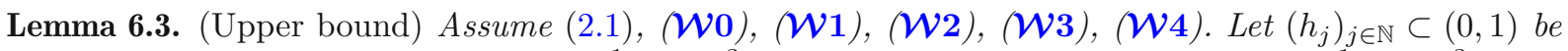
a vanishing sequence. For every $\mathbf{v} \in W^{1, p}\left(\Omega, \mathbb{R}^{3}\right)$, there exists a sequence $\left(\mathbf{v}_{j}\right)_{j \in \mathbb{N}} \subset W^{1, p}\left(\Omega, \mathbb{R}^{3}\right)$ such that $\mathbf{v}_{j} \rightarrow \mathbf{v}$ weakly in $W^{1, p}\left(\Omega, \mathbb{R}^{3}\right)$ as $j \rightarrow+\infty$ and

$$
\limsup _{j \rightarrow+\infty} \mathcal{F}_{h_{j}}^{I}\left(\mathbf{v}_{j}\right) \leq \mathcal{E}^{I}(\mathbf{v})
$$


Proof. It is enough to prove the result in case $\mathbf{v} \in H_{\text {div }}^{1}(\Omega)$. Let us define $\mathcal{E}: H^{1}\left(\Omega, \mathbb{R}^{3}\right) \rightarrow \mathbb{R}$ as

$$
\mathcal{E}(\mathbf{u}):=\frac{1}{2} \int_{\Omega} \mathbb{E}(\mathbf{u}) D^{2} \mathcal{W}(x, \mathbf{I}) \mathbb{E}(\mathbf{u}) \mathrm{d} x-\mathcal{L}(\mathbf{u}) .
$$

We take the sequence $\left(\mathbf{v}_{j}\right)_{j \in \mathbb{N}}$ from Lemma 6.2. Property (ii) of Lemma 6.2 yields $\mathbf{I}+h_{j} \nabla \mathbf{v}_{j} \in \mathcal{U}$ for a.e. $x$ in $\Omega$ if $j$ is large enough, where $\mathcal{U}$ is the neighbor of $S O(3)$ that appears in $(\mathcal{W} 3)$. In particular, $D^{2} \mathcal{W}(x, \cdot) \in C^{2}(\mathcal{U})$ for a.e. $x \in \Omega$ and we make use of (3.6) together with $\operatorname{det}\left(\mathbf{I}+h_{j} \nabla \mathbf{v}_{j}\right)=1$ to obtain

$$
\begin{aligned}
\limsup _{j \rightarrow+\infty}\left|\mathcal{F}_{h_{j}}^{I}\left(\mathbf{v}_{j}\right)-\mathcal{E}\left(\mathbf{v}_{j}\right)\right| & \leq \limsup _{j \rightarrow+\infty} \int_{\Omega}\left|\frac{1}{h_{j}^{2}} \mathcal{W}^{I}\left(x, \mathbf{I}+h_{j} \nabla \mathbf{v}_{j}\right)-\frac{1}{2} \nabla \mathbf{v}_{j}^{T} D^{2} \mathcal{W}(x, \mathbf{I}) \nabla \mathbf{v}_{j}\right| \mathrm{d} x \\
& =\limsup _{j \rightarrow+\infty} \int_{\Omega}\left|\frac{1}{h_{j}^{2}} \mathcal{W}\left(x, \mathbf{I}+h_{j} \nabla \mathbf{v}_{j}\right)-\frac{1}{2} \nabla \mathbf{v}_{j}^{T} D^{2} \mathcal{W}(x, \mathbf{I}) \nabla \mathbf{v}_{j}\right| \mathrm{d} x \\
& \leq \limsup _{j \rightarrow+\infty} \int_{\Omega} \omega\left(h_{j}\left|\nabla \mathbf{v}_{j}\right|\right)\left|\nabla \mathbf{v}_{j}\right|^{2} \mathrm{~d} x \\
& \leq \limsup _{j \rightarrow+\infty}\left\|\omega\left(h_{j} \nabla \mathbf{v}_{j}\right)\right\|_{L^{\infty}(\Omega)} \int_{\Omega}\left|\nabla \mathbf{v}_{j}\right|^{2} \mathrm{~d} x=0 .
\end{aligned}
$$

The limit in the last line is zero since $h_{j} \nabla \mathbf{v}_{j} \rightarrow 0$ in $L^{\infty}(\Omega)$, since $\omega$ is increasing with $\lim _{t \rightarrow 0^{+}} \omega(t) \rightarrow 0$ and since $\left(\mathbf{v}_{j}\right)_{j \in \mathbb{N}}$ is converging in $H^{1}(\Omega)$ as $j \rightarrow+\infty$ by Lemma 6.2 . But the $H^{1}(\Omega)$ convergence also entails $\mathcal{E}\left(\mathbf{v}_{j}\right) \rightarrow \mathcal{E}(\mathbf{v})$ as $j \rightarrow+\infty$. Hence,

$$
\begin{aligned}
\limsup _{j \rightarrow+\infty}\left|\mathcal{F}_{h_{j}}^{I}\left(\mathbf{v}_{j}\right)-\mathcal{E}^{I}(\mathbf{v})\right| & =\limsup _{j \rightarrow+\infty}\left|\mathcal{F}_{h_{j}}^{I}\left(\mathbf{v}_{j}\right)-\mathcal{E}(\mathbf{v})\right| \\
& \leq \limsup _{j \rightarrow+\infty}\left|\mathcal{F}_{h_{j}}^{I}\left(\mathbf{v}_{j}\right)-\mathcal{E}\left(\mathbf{v}_{j}\right)\right|+\limsup _{j \rightarrow+\infty}\left|\mathcal{E}\left(\mathbf{v}_{j}\right)-\mathcal{E}(\mathbf{v})\right|=0 .
\end{aligned}
$$

Therefore, along the sequence $\left(\mathbf{v}_{j}\right)_{j \in \mathbb{N}}$ provided by Lemma 6.2 , we get $\mathcal{F}_{h_{j}}^{I}\left(\mathbf{v}_{j}\right) \rightarrow \mathcal{E}^{I}(\mathbf{v})$ as $j \rightarrow+\infty$. The result is proven.

\section{Convergence of minimizers}

We show that $\mathcal{E}^{I}$ and $\mathcal{F}^{I}$ have the same minimizers thus concluding the proof of the main result.

Lemma 7.1. Assume $(2.1),(\mathcal{L} 1),(\mathcal{L} 2),(\mathcal{W} \mathbf{0}),(\mathcal{W} 1),(\mathcal{W} \mathbf{2}),(\mathcal{W} \mathbf{3}),(\mathcal{W} 4)$. On $W^{1, p}\left(\Omega, \mathbb{R}^{3}\right)$ we have

$$
\min \mathcal{F}^{I}=\min \mathcal{E}^{I}
$$

and

$$
\operatorname{argmin} \mathcal{F}^{I}=\operatorname{argmin} \mathcal{E}^{I} .
$$

Proof. Existence of minimizers of $\mathcal{E}^{I}$ on $W^{1, p}\left(\Omega, \mathbb{R}^{3}\right)$ follows by standard arguments. Indeed, (3.5) and (3.11) imply that a minimizing sequence $\left(\mathbf{u}_{n}\right)_{n \in \mathbb{N}} \subset H_{\text {div }}^{1}\left(\Omega, \mathbb{R}^{3}\right)$ of $\mathcal{E}^{I}$ satisfies $\sup _{n \in \mathbb{N}}\left\|\mathbb{E}\left(\mathbf{u}_{n}\right)\right\|_{L^{2}(\Omega)}<$ $+\infty$, and Lemma 3.1 entails the existence of $\mathbf{u} \in H^{1}\left(\Omega, \mathbb{R}^{3}\right)$ such that up to subsequences $\mathbb{E}\left(\mathbf{u}_{n}\right) \rightarrow \mathbb{E}(\mathbf{u})$ weakly in $L^{2}\left(\Omega, \mathbb{R}^{3 \times 3}\right)$. By $(3.10)$ and $(\mathcal{L} 1)$, we deduce $\mathcal{L}\left(\mathbf{u}_{n}\right) \rightarrow \mathcal{L}(\mathbf{u})$, up to subsequences, as $n \rightarrow+\infty$. By the weak $L^{2}(\Omega)$ lower semicontinuity of $\mathbf{F} \mapsto \int_{\Omega} \mathbf{F}^{T} D^{2} \mathcal{W}(x, \mathbf{I}) \mathbf{F} \mathrm{d} x$, we deduce that $\mathbf{u}$ is a minimizer of $\mathcal{E}^{I}$ over $W^{1, p}\left(\Omega, \mathbb{R}^{3}\right)$. Moreover, first order minimality conditions show that all the minimizers of $\mathcal{E}^{I}$ have the same infinitesimal strain tensor. In particular, minimizers of $\mathcal{E}^{I}$ are unique up to rigid displacements. 
By taking into account that $\mathcal{F}^{I}(\mathbf{v}) \leq \mathcal{E}^{I}(\mathbf{v})$ for every $\mathbf{v} \in H^{1}(\Omega)$, and setting $\mathbf{z}_{\mathbf{W}}(x):=\frac{1}{2} \mathbf{W}^{2} x$ for every $\mathbf{W} \in \mathbb{R}_{\text {skew }}^{3 \times 3}$, we get $\mathbb{E}\left(\mathbf{z}_{\mathbf{W}}\right)=\frac{1}{2} \mathbf{W}^{2}$ and

$$
\begin{aligned}
\min _{\mathbf{v} \in H^{1}(\Omega)} \mathcal{E}^{I}(\mathbf{v}) & \geq \inf _{\mathbf{v} \in H^{1}(\Omega)} \mathcal{F}^{I}(\mathbf{v})=\inf _{\mathbf{v} \in H^{1}(\Omega)}\left\{\min _{\mathbf{w} \in \mathbb{R}_{\text {skew }}^{3 \times 3}}\left\{\int_{\Omega} \mathcal{Q}^{I}\left(x, \mathbb{E}(\mathbf{v})-\frac{1}{2} \mathbf{W}^{2}\right) \mathrm{d} x-\mathcal{L}(\mathbf{v})\right\}\right\} \\
& =\min _{\mathbf{W} \in \mathbb{R}_{\text {skew }}^{3 \times 3}}\left\{\min _{\mathbf{v} \in H^{1}(\Omega)}\left\{\int_{\Omega} \mathcal{Q}^{I}\left(x, \mathbb{E}(\mathbf{v})-\frac{1}{2} \mathbf{W}^{2}\right) \mathrm{d} x-\mathcal{L}(\mathbf{v})\right\}\right\} \\
& =\min _{\mathbf{W} \in \mathbb{R}_{\text {skew }}^{3 \times 3}}\left\{\min _{\mathbf{v} \in H^{1}(\Omega)}\left\{\int_{\Omega} \mathcal{Q}^{I}\left(x, \mathbb{E}\left(\mathbf{v}-\mathbf{z}_{\mathbf{W}}\right)\right) \mathrm{d} x-\mathcal{L}\left(\mathbf{v}-\mathbf{z}_{\mathbf{W}}\right)-\mathcal{L}\left(\mathbf{z}_{\mathbf{W}}\right)\right\}\right\} \\
& =\min _{\mathbf{z} \in H^{1}(\Omega)} \mathcal{E}^{I}(\mathbf{z})-\max _{\mathbf{W} \in \mathbb{R}_{\text {skew }}^{3 \times 3}} \mathcal{L}\left(\mathbf{z}_{\mathbf{W}}\right) \geq \min _{H^{1}(\Omega)} \mathcal{E}^{I}
\end{aligned}
$$

where last inequality follows by $\mathcal{L}\left(z_{\mathbf{W}}\right) \leq 0$. Therefore, also $\min \mathcal{F}^{I}$ exists on $W^{1, p}\left(\Omega, \mathbb{R}^{3}\right)$ and $(7.1)$ is proved so we are left to show (7.2).

First assume $\mathbf{v} \in \operatorname{argmin} \mathcal{F}^{I}$ and let

$$
\mathbf{W}_{\mathbf{v}} \in \operatorname{argmin}\left\{\int_{\Omega} \mathcal{Q}^{I}\left(x, \mathbb{E}(\mathbf{v})-\frac{1}{2} \mathbf{W}^{2}\right) \mathrm{d} x: \mathbf{W} \in \mathbb{R}_{\text {skew }}^{3 \times 3}\right\} .
$$

If $\mathbf{W}_{\mathbf{v}} \neq \mathbf{0}$ then, by setting $\mathbf{z}_{\mathbf{W}_{\mathbf{v}}}(x)=\frac{1}{2} \mathbf{W}_{\mathbf{v}}^{2} x$ we get $\mathbb{E}\left(\mathbf{z}_{\mathbf{W}_{\mathbf{v}}}\right)=\nabla \mathbf{z}_{\mathbf{W}_{\mathbf{v}}}=\frac{1}{2} \mathbf{W}_{\mathbf{v}}^{2}$ and, by compatibility $(\mathcal{L} \mathbf{2})$ we obtain

$$
\begin{aligned}
& \min \mathcal{F}^{I}=\mathcal{F}^{I}(\mathbf{v})=\int_{\Omega} \mathcal{Q}^{I}\left(x, \mathbb{E}\left(\mathbf{v}-\mathbf{z}_{\mathbf{W}_{\mathbf{v}}}\right)\right) \mathrm{d} x-\mathcal{L}\left(\mathbf{v}-\mathbf{z}_{\mathbf{W}_{\mathbf{v}}}\right)-\mathcal{L}\left(\mathbf{z}_{\mathbf{W}_{\mathbf{v}}}\right)= \\
& \mathcal{E}^{I}\left(\mathbf{v}-\mathbf{z}_{\mathbf{W}_{\mathbf{v}}}\right)-\mathcal{L}\left(\mathbf{z}_{\mathbf{W}_{\mathbf{v}}}\right) \geq \min \mathcal{E}^{I}-\mathcal{L}\left(\mathbf{z}_{\mathbf{W}_{\mathbf{v}}}\right)>\min \mathcal{E}^{I},
\end{aligned}
$$

a contradiction. Therefore, $\mathbf{W}_{\mathbf{v}}=\mathbf{0}, \mathbf{z}_{\mathbf{W}_{\mathbf{v}}}=\mathbf{0}$, and all the inequalities in (7.4) turn out to be equalities, hence we get $\mathcal{F}^{I}(\mathbf{v})=\mathcal{E}^{I}(\mathbf{v})=\min \mathcal{E}^{I}=\min \mathcal{F}^{I}$, therefore $\mathbf{v} \in \operatorname{argmin} \mathcal{E}$ and $\operatorname{argmin} \mathcal{F}^{I} \subseteq \operatorname{argmin} \mathcal{E}^{I}$. In order to show the opposite inclusion, we assume $\mathbf{v} \in \operatorname{argmin} \mathcal{E}^{I}$ and still referring to the choice (7.3) we get $2 \operatorname{div} \mathbf{v}=0=\operatorname{Tr} \mathbf{W}_{\mathbf{v}}^{2}=-\left|\mathbf{W}_{\mathbf{v}}\right|^{2}$. Therefore, $\mathcal{E}^{I}(\mathbf{v})=\mathcal{F}^{I}(\mathbf{v})$ and $\mathbf{v} \in \operatorname{argmin} \mathcal{F}^{I}$.

Remark 7.2. The proof of Lemma 7.1 shows that, although Theorem 2.1 is not true if $(\mathcal{L} 2)$ is replaced by the weaker condition

$$
\mathcal{L}\left(\mathbf{W}^{2} x\right) \leq 0 \quad \forall \mathbf{W} \in \mathbb{R}_{\text {skew }}^{3 \times 3},
$$

still $\mathcal{E}^{I}$ and $\mathcal{F}^{I}$ have the same minimal values under such weaker condition. As an example, we may consider $\mathbf{f}$ and $\mathbf{g}$ as in Remark 2.8, but with $\int_{\Omega} \phi(x) \mathrm{d} x=\lambda|\Omega|$ instead of $\int_{\Omega} \phi(x) \mathrm{d} x<\lambda|\Omega|$. In this case, $\mathcal{L}\left(\mathbf{W}^{2} x\right)=0$ for any $\mathbf{W} \in \mathbb{R}_{\text {skew }}^{3 \times 3}$ and then Theorem 2.1 does not apply (see Remark 2.5). However, still $\mathcal{E}^{I}(\mathbf{v})$ is minimal (with minimal value 0 ) if and only if $\mathbf{v}$ is a rigid displacement. Moreover, $\mathcal{F}^{I}$ is minimal on rigid displacements as well.

Proof of Theorem 2.1. We obtain (2.5) from Lemma 4.1. If $\left(\mathbf{v}_{j}\right)_{j \in \mathbb{N}} \subset W^{1, p}\left(\Omega, \mathbb{R}^{3}\right)$ is a sequence such that

$$
\lim _{j \rightarrow+\infty}\left(\mathcal{F}_{h_{j}}^{I}\left(\mathbf{v}_{j}\right)-\inf _{W^{1, p}\left(\Omega, \mathbb{R}^{3}\right)} \mathcal{F}_{h_{j}}^{I}\right)=0
$$


then by Lemma 4.2 there exists $\mathbf{v}_{*} \in H^{1}(\Omega)$ such that, up to subsequences, $\mathbb{E}\left(\mathbf{v}_{j}\right) \rightarrow \mathbb{E}\left(\mathbf{v}_{*}\right)$ weakly in $L^{p}(\Omega)$ hence by Lemma 5.9

$$
\liminf _{j \rightarrow+\infty} \mathcal{F}_{h_{j}}^{I}\left(\mathbf{v}_{j}\right) \geq \mathcal{F}^{I}\left(\mathbf{v}_{*}\right) .
$$

On the other hand by Lemma 6.3 for every $\mathbf{v} \in H^{1}(\Omega)$, there exists a sequence $\left(\mathbf{v}_{j}\right)_{j \in \mathbb{N}} \subset W^{1, p}(\Omega)$ such that $\mathbb{E}\left(\mathbf{v}_{j}\right) \rightarrow \mathbb{E}\left(\mathbf{v}_{*}\right)$ weakly in $L^{p}(\Omega)$ and

$$
\limsup _{j \rightarrow+\infty} \mathcal{F}_{h_{j}}^{I}\left(\mathbf{v}_{j}\right) \leq \mathcal{E}^{I}(\mathbf{v})
$$

that is, $\mathcal{F}^{I}\left(\mathbf{v}_{*}\right) \leq \mathcal{E}^{I}(\mathbf{v})$ for every $\mathbf{v} \in H^{1}(\Omega)$. Hence, $\mathcal{F}^{I}\left(\mathbf{v}_{*}\right) \leq \min _{W^{1, p}(\Omega)} \mathcal{E}^{I}$ and by Lemma 7.1 we obtain $\mathcal{F}^{I}\left(\mathbf{v}_{*}\right) \leq \min _{W^{1, p}(\Omega)} \mathcal{E}^{I}=\min _{W^{1, p}(\Omega)} \mathcal{F}^{I}$ so that by $(7.5),(7.6)$

$$
\lim _{j \rightarrow+\infty} \mathcal{F}_{h_{j}}^{I}\left(\mathbf{v}_{j}\right)=\mathcal{F}^{I}\left(\mathbf{v}_{*}\right)=\min _{W^{1, p}(\Omega)} \mathcal{F}^{I}=\min _{W^{1, p}(\Omega)} \mathcal{E}^{I}
$$

thus completing the proof.

\section{Acknowledgements}

Open access funding provided by Università degli Studi di Genova within the CRUI-CARE Agreement. The authors acknowledge support from the MIUR-PRIN Project No. 2017TEXA3H. The authors are members of the GNAMPA group of the Istituto Nazionale di Alta Matematica (INdAM).

Open Access. This article is licensed under a Creative Commons Attribution 4.0 International License, which permits use, sharing, adaptation, distribution and reproduction in any medium or format, as long as you give appropriate credit to the original author(s) and the source, provide a link to the Creative Commons licence, and indicate if changes were made. The images or other third party material in this article are included in the article's Creative Commons licence, unless indicated otherwise in a credit line to the material. If material is not included in the article's Creative Commons licence and your intended use is not permitted by statutory regulation or exceeds the permitted use, you will need to obtain permission directly from the copyright holder. To view a copy of this licence, visit http://creativecommons.org/licenses/by/4.0/.

Publisher's Note Springer Nature remains neutral with regard to jurisdictional claims in published maps and institutional affiliations.

\section{References}

[1] Agostiniani, V., Blass, T., Koumatos, K.: From nonlinear to linearized elasticity via Gamma-convergence: the case of multiwell energies satisfying weak coercivity conditions. Math. Models Methods Appl. Sci. 25, 1-38 (2015)

[2] Agostiniani, V., Dal Maso, G., De Simone, A.: Linear elasticity obtained from finite elasticity by Gamma-convergence under weak coerciveness conditions. Ann. Inst. H. Poincaré Anal. non Linéaire 29, 715-735 (2012)

[3] Alicandro, R., Dal Maso, G., Lazzaroni, G., Palombaro, M.: Derivation of a linearised elasticity model from singularly perturbed multiwell energy functionals. Arch. Ration. Mech. Anal. 230, 1-45 (2018)

[4] Anzellotti, G., Baldo, S., Percivale, D.: Dimension reduction in variational problems, asymptotic development in $\Gamma$ convergence and thin structures in elasticity. Asympt. Anal. 9, 61-100 (1994)

[5] Arruda, E.M., Boyce, M.C.: A three-dimensional constitutive model for the large stretch behavior of rubber elastic materials. J. Mech. Phys. Solids 41(2), 389-412 (1993)

[6] Boyce, M.C., Arruda, E.M.: Constitutive models of rubber elasticity: a review. Rubber Chem. Technol. 73(3), 504-523 (2000)

[7] Chaves, E.W.: Notes on Continuum Mechanics. Springer, Berlin (2013)

[8] Ciarlet, P.G.: Mathematical Elasticity. Volume I: Three-Dimensional Elasticity. Elsevier, Amsterdam (1988)

[9] Chuong, C.J., Fung, Y.C.: Compressibility and constitutive equation of arterial wall in radial compression experiments. J. Biomech. 17(1), 35-40 (1984)

[10] Conti, S., Dolzmann, G.: Г-convergence for incompressible elastic plates. Calc. Var. 34, 531-551 (2009) 
[11] Dal Maso, G., Negri, M., Percivale, D.: Linearized elasticity as $\Gamma$-limit of finite elasticity. Set Valued Anal. 10(2-3), 165-183 (2002)

[12] Friesecke, G., James, R.D., Müller, S.: A theorem on geometric rigidity and the derivation of non linear plate theory from three dimensional elasticity. Commun. Pure Appl. Math. 55, 1461-1506 (2002)

[13] Friesecke, G., James, R.D., Müller, S.: A hierarchy of plate models from nonlinear elasticity by Gamma-convergence. Arch. Ration. Mech. Anal. 1(80), 183-236 (2006)

[14] Gurtin, M.E.: An Introduction to Continuum Mechanics. Academic Press, New York (1981)

[15] Henrot, A., Pierre, M.: Shape Variation and Optimization: A Geometrical Analysis. EMS Tracts in Mathematics. European Mathematical Society, Zurich (2018)

[16] Holzapfel, G.A.: Nonlinear Solid Mechanics: A Continuum Approach for Engineering. Wiley, Chichester (2000)

[17] Holzapfel, G.A., Ogden, R.E. (eds.): Biomechanics of Soft Tissue in Cardiovascular System. Springer, New York (2003)

[18] Jesenko, M., Schmidt, B.: Geometric linearization of theories for incompressible elastic materials and applications. Preprint arXiv:2004.11271

[19] Kalita, P., Schaefer, R.: Mechanical models of artery walls. Arch. Comput. Methods Eng. 15, 1-36 (2008)

[20] Kato, T., Mitrea, M., Ponce, G., Taylor, M.: Extension and representation of divergence-free vector fields on bounded domains. Math. Res. Lett. 7, 643-650 (2000)

[21] Lecumberry, M., Müller, S.: Stability of slender bodies under compression and validity of von Kármán theory. Arch. Ration. Mech. Anal. 193, 255-310 (2009)

[22] Love, A.E.: A Treatise on the Mathematical Theory of Elasticity. Dover, New York (1944)

[23] Maddalena, F., Percivale, D., Tomarelli, F.: A new variational approach to linearization of traction problems in elasticity. J. Optim. Theory Appl. 182, 383-403 (2019)

[24] Maddalena, F., Percivale, D., Tomarelli, F.: The gap in pure traction problems between linear elasticity and variational limit of finite elasticity. Arch. Ration. Mech. Anal. 234, 1091-1120 (2019)

[25] Mainini, E., Percivale, D.: Linearization of elasticity models for incompressible materials. Preprint arXiv:2004.09286

[26] Marckmann, G., Verron, E.: Comparison of hyperelastic models for rubberlike materials. Rubber Chem. Technol. 79(5), $835-858(2006)$

[27] Nitsche, J.A.: On Korn's second inequality. RAIRO Anal. Numér. 15, 237-248 (1981)

[28] Ogden, R.W.: Mechanics of Rubberlike Solids. In: Gutkowski, W., Kowalewski, T.A. (eds.) Mechanics of the 21st Century. Springer, Dordrecht (2005)

[29] Ogden, R.W.: Non-linear Elastic Deformations. Dover, New York (1997)

[30] Ogden, R.W.: Recent advances in the phenomenological theory of rubber elasticity. Rubber Chem. Technol. 59, 361-383 (1986)

[31] Percivale, D., Tomarelli, F.: Scaled Korn-Poincaré inequality in BD and a model of elastic plastic cantilever. Asymptot. Anal. 23(3-4), 291-311 (2000)

[32] Percivale, D., Tomarelli, F.: From SBD to SBH: the elastic-plastic plate. Interfaces Free Bound. 4(2), 137-165 (2002)

[33] Percivale, D., Tomarelli, F.: A variational principle for plastic hinges in a beam. Math. Models Methods Appl. Sci. 19(12), 2263-2297 (2009)

[34] Percivale, D., Tomarelli, F.: Smooth and broken minimizers of some free discontinuity problems. In: Colli, P., et al. (eds.) Solvability, Regularity, and Optimal Control of Boundary Value Problems for PDEs. Springer INdAM Series, vol. 22, pp. 431-468. Springer, Berlin (2017)

[35] Podio-Guidugli, P.: On the validation of theories of thin elastic structures. Meccanica 49(6), 1343-1352 (2014)

[36] Saccomandi, G., Ogden, R.W. (eds.): Mechanics and Thermomechanics of Rubberlike Solids. CISM Courses and Lectures, vol. 452. Springer, Wien (2004)

[37] Schmidt, B.: Linear Gamma-limits of multiwell energies in nonlinear elasticity theory. Contin. Mech. Thermodyn. 20(6), 375-396 (2008)

[38] Slaughter, W.S.: The Linearized Theory of Elasticity. Birkhäuser, Basel (2002)

[39] Steinmann, P., Hossain, M., Possart, G.: Hyperelastic models for rubber-like materials: consistent tangent operators and suitability for Treloar's data. Arch. Appl. Mech. 82, 1183-1217 (2012)

[40] Yeoh, O.H.: Characterization of elastic properties of carbon-black-filled rubber vulcanizates. Rubber Chem. Technol. 63(5), 792-805 (1990)

[41] Yeoh, O.H.: Some forms of the strain energy function for rubber. Rubber Chem. Technol. 66(5), 754-771 (1993) 
Edoardo Mainini and Danilo Percivale

Dipartimento di Ingegneria meccanica, energetica, gestionale e dei trasporti

Università degli studi di Genova

Via all'Opera Pia, 15

16145 Genova

Italy

Edoardo Mainini

e-mail: mainini@dime.unige.it

URL: http://www.dime.unige.it/it/users/edoardo-mainini

Danilo Percivale

e-mail: percivale@dime.unige.it

(Received: June 11, 2020; revised: July 27, 2020) 